\title{
Protein kinase N2 mediates flow-induced endothelial NOS activation and vascular tone regulation
}

\author{
Young-June Jin, ${ }^{1}$ Ramesh Chennupati, ${ }^{1}$ Rui Li, ${ }^{1}$ Guozheng Liang, ${ }^{1}$ ShengPeng Wang, ${ }^{1,2}$ András Iring, ${ }^{1,3}$ Johannes Graumann, ${ }^{4}$ \\ Nina Wettschureck, ${ }^{1,5,6,7}$ and Stefan Offermanns s,5,6,7 $^{15}$ \\ 'Department of Pharmacology, Max Planck Institute for Heart and Lung Research, Bad Nauheim, Germany. '2ardiovascular Research Center, School of Basic Medical Sciences, Xi'an Jiaotong University Health \\ Science Center, Yanta District, Xi'an, China. ${ }^{3}$ Laboratory of Molecular Medicine, Institute of Experimental Medicine, Hungarian Academy of Sciences, Budapest, Hungary. ${ }^{4}$ Scientific Service Group Biomolecular \\ Mass Spectrometry, Max Planck Institute for Heart and Lung Research, Bad Nauheim, Germany. ${ }^{5}$ Centre for Molecular Medicine, Medical Faculty, JW Goethe University Frankfurt, Frankfurt, Germany. \\ ${ }^{6}$ Cardiopulmonary Institute (CPI), Frankfurt, Germany. ${ }^{7}$ Cerman Center for Cardiovascular Research (DZHK), Rhine-Main Site, Frankfurt and Bad Nauheim, Germany.
}

\begin{abstract}
Formation of NO by endothelial NOS (eNOS) is a central process in the homeostatic regulation of vascular functions including blood pressure regulation, and fluid shear stress exerted by the flowing blood is a main stimulus of eNOS activity. Previous work has identified several mechanosensing and -transducing processes in endothelial cells, which mediate this process and induce the stimulation of eNOS activity through phosphorylation of the enzyme via various kinases including AKT. How the initial mechanosensing and signaling processes are linked to eNOS phosphorylation is unclear. In human endothelial cells, we demonstrated that protein kinase N2 (PKN2), which is activated by flow through the mechanosensitive cation channel Piezo1 and $\mathrm{G}_{q} / \mathrm{C}_{11}$-mediated signaling, as well as by $\mathrm{Ca}^{2+}$ and phosphoinositide-dependent protein kinase 1 (PDK1), plays a pivotal role in this process. Active PKN2 promoted the phosphorylation of human eNOS at serine 1177 and at a newly identified site, serine 1179. These phosphorylation events additively led to increased eNOS activity. PKN2-mediated eNOS phosphorylation at serine 1177 involved the phosphorylation of AKT synergistically with mTORC2-mediated AKT phosphorylation, whereas active PKN2 directly phosphorylated human eNOS at serine 1179. Mice with induced endothelium-specific deficiency of PKN2 showed strongly reduced flow-induced vasodilation and developed arterial hypertension accompanied by reduced eNOS activation. These results uncover a central mechanism that couples upstream mechanosignaling processes in endothelial cells to the regulation of eNOS-mediated NO formation, vascular tone, and blood pressure.
\end{abstract}

\section{Introduction}

Endothelial cells are located at the interface between the blood and the vessel wall and are centrally involved in autoregulatory processes, which control vascular tone and homeostasis. NO produced by endothelial NOS (eNOS) plays a critical role in these regulatory processes by promoting vascular relaxation and modulating vascular inflammation and adaptation to physiological and pathophysiological stimuli (1-3). The activity of eNOS is regulated by transcriptional and posttranscriptional mechanisms. The regulation of eNOS activity by fluid shear stress exerted by flowing blood is one of the major mechanisms controlling basal activity of eNOS. Multiple studies have shown that eNOS-mediated NO formation in response to sustained flow involves phosphorylation of the enzyme (3-5) and the 2 best-described residues of human eNOS phosphorylated in response to flow are serines 633 and 1177 $(4,6-9)$. Protein kinase A (PKA) has been reported to be the major enzyme mediating the phosphorylation of serine $633(8,10-12)$, and AKT has been described as the major protein kinase responsible for flow-induced phosphorylation of serine 1177 (6, 7). Later,

Conflict of interest: The authors have declared that no conflict of interest exists. Copyright: (5) 2021, American Society for Clinical Investigation.

Submitted: November 4, 2020; Accepted: September 1, 2021; Published: November 1, 2021 Reference information: J Clin Invest. 2021;131(21):e145734.

https://doi.org/10.1172/JCl145734. other related protein kinases, including AMPK and PKA, have also been reported to be able to mediate flow-induced eNOS phosphorylation at serine $1177(4,5,13,14)$.

Flow-induced regulation of AKT-dependent eNOS phosphorylation is mediated by the endothelial mechanosensitive cation channel Piezo1, which is activated by fluid shear stress and promotes the release of ATP from endothelial cells, resulting in activation of the $G_{q} / G_{11}$-coupled purinergic $P 2 Y_{2}$ receptor (15). Receptor activation induces downstream signaling processes, resulting in the stimulation of AKT activity and the subsequent phosphorylation of human eNOS at serine 1177, which then leads to increased NO formation (16). An important role of AKT1 in flow-induced eNOS phosphorylation and activation has been indicated by in vitro-knockdown studies (17) as well as in endothelium-specific AKT1-deficient mice, which show reduced phosphorylation of the corresponding serine residue of mouse eNOS, reduced circulating NO levels, and increased blood pressure (18). How the upstream mechanosignaling processes are linked to AKT-dependent eNOS activation is not clear, however. It has been shown that phosphoinositide 3-kinase (PI3K) activity plays a role upstream of AKT also in flow-induced endothelial processes $(6,7,19)$. However, maximal activation of AKT requires not only binding of the PI3K product phosphatidyl-inositol-3,4,5-trisphosphate ( $\mathrm{PIP}_{3}$ ) to AKT but also phosphorylation of the enzyme at threonine 308 by phosphoinositide-dependent protein kinase 1 (PDK1) as well as at 
A

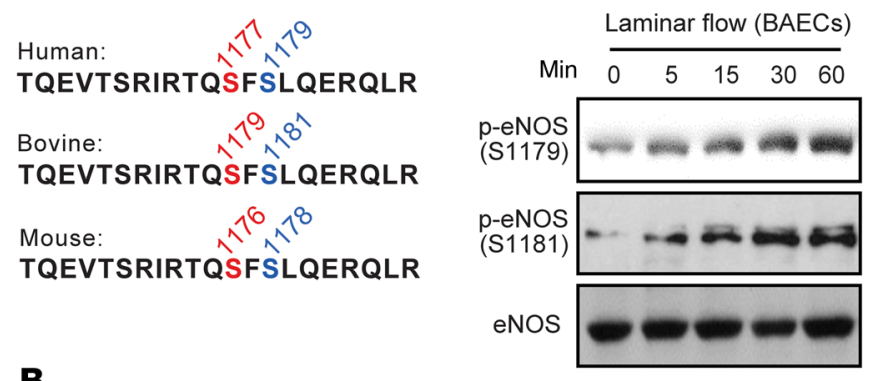

D

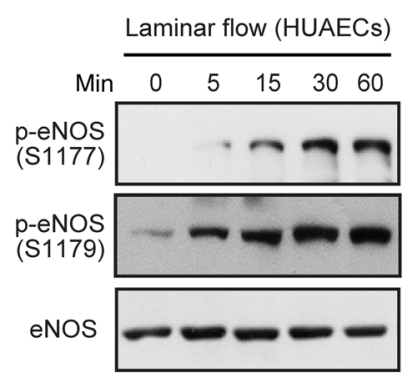

E

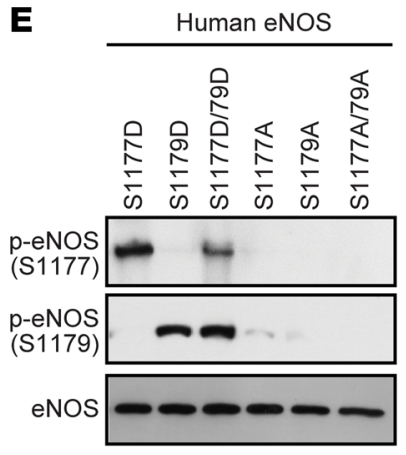

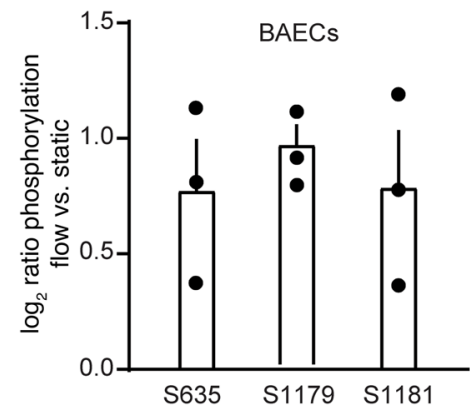

$\mathbf{F}$

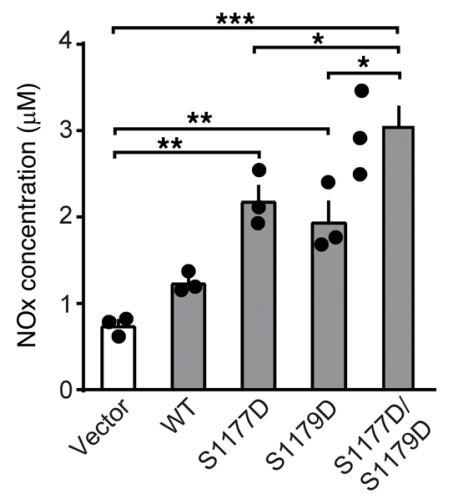

Lenti-eNOS

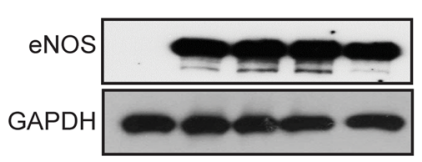

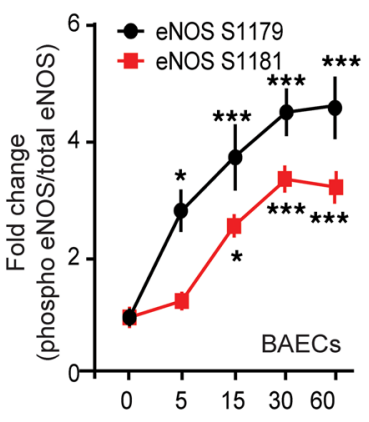

G

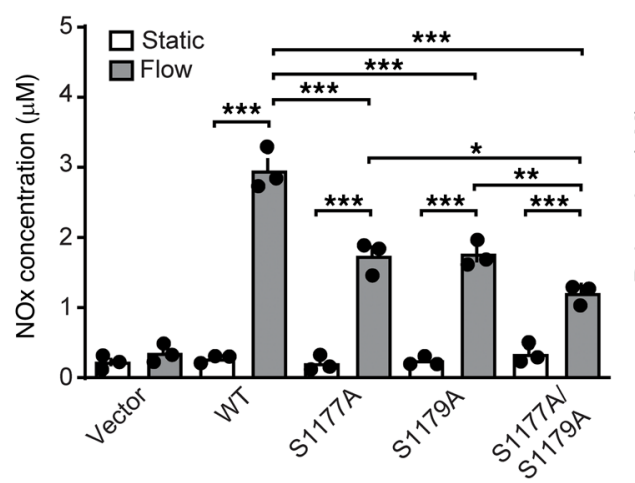

Lenti-eNOS

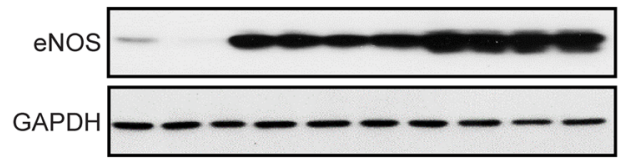

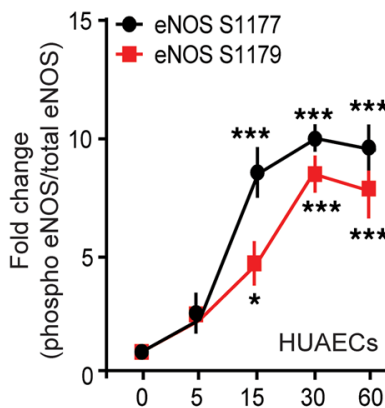

Human eNOS
\begin{tabular}{|c|c|c|c|c|c|c|}
\hline WT & T & Q & S & F & S & L \\
\hline S1177A & T & Q & A & F & S & L \\
\hline S1177D & T & Q & D & F & S & L \\
\hline S1179A & T & O & S & F & A & L \\
\hline S1179D & T & Q & S & F & D & L \\
\hline S1177A/79A & T & Q & A & F & A & L \\
\hline S1177D/79D & T & Q & D & F & D & L \\
\hline
\end{tabular}

\section{H}

H - ENOS KO+EGFP

$407-\mathrm{eNOS} \mathrm{KO}+\mathrm{WT}$

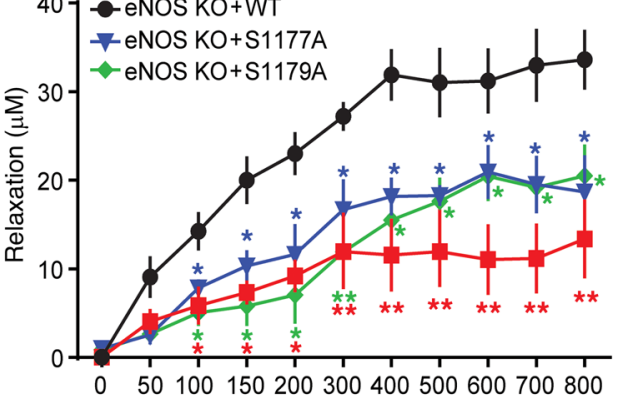

Flow ( $\mu \mathrm{L} / \mathrm{min})$

eNOS-KO

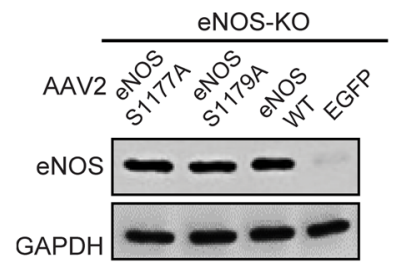

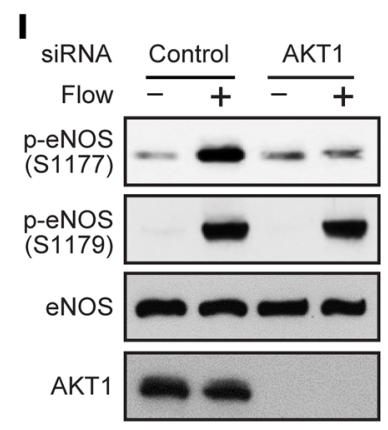
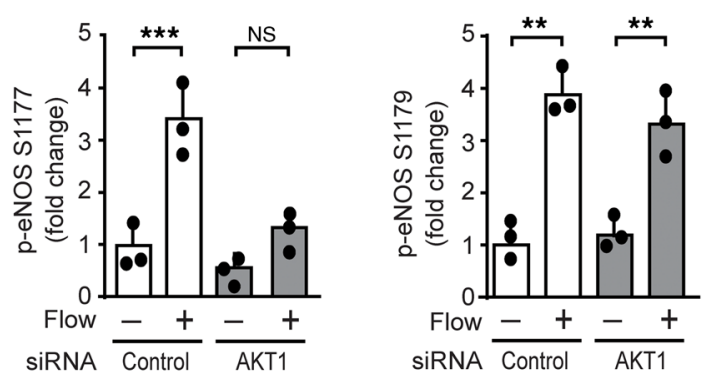
Figure 1. Flow induces eNOS phosphorylation at serines 1177 and 1179. (A) eNOS amino acid sequence in different species. (B) Phosphorylation of eNOS serine residues of BAECs exposed to laminar flow (15 dynes/cm²) determined by LC-MS/MS. Shown are the fold changes of the corrected ratios of flow versus static conditions $(n=3)$. (C and D) BAECs (C) and HUAECs (D) were exposed to laminar flow (15 dynes $\left./ \mathrm{cm}^{2}\right)$, and total and p-eNOS levels were determined by immunoblotting. Graphs show the densitometric evaluation ( $n=3$ independent experiments). (E) HEK293 cells expressing the indicated mutants of human eNOS were lysed and analyzed by immunoblotting using phosphosite-specific antibodies. ( $F$ and $\mathbf{G}$ ) WT or mutant human eNOS was expressed by lentiviral transduction after siRNA-mediated eNOS knockdown in HUAECs, and the NOx concentration in the cell culture medium was determined in the absence ( $\mathbf{F}$ and $\mathbf{G}$ ) or presence (G) of flow. Expression of eNOS was analyzed by immunoblotting ( $\mathbf{F}$ and $\mathbf{G}$, lower panels). $n=3$ independent experiments. (H) eNOS ${ }^{-1-}$ mice were infected with AAV2-QuadYF virus, which transduces WT or mutant eNOS or EGFP. Seven days later, carotid arteries were isolated, and flow-induced vasorelaxation was analyzed. The immunoblot demonstrates equal expression levels of different eNOS mutants. (I) HUAECs transfected with control siRNA or siRNAs directed against AKT1 were exposed to laminar flow $\left(15 \mathrm{dynes} / \mathrm{cm}^{2}\right)$. Total AKT and total and p-eNOS levels were determined by immunoblotting. Graphs show the densitometric evaluation of blots ( $n=3$ independent experiments). Data represent the mean \pm SEM. ${ }^{*} P \leq 0.05,{ }^{*} P \leq 0.01$, and ${ }^{* *} P \leq 0.001$, by 2 -way ANOVA with Bonferroni's post-hoc test (C, D, $\mathbf{G}$, and $\mathbf{H} ; P$ values in $\mathbf{H}$ describe the difference compared with eNOS WT) and 1-way ANOVA, with Tukey's post hoc test ( $\mathbf{F}$ and I). $P$ values in $1 c$ and d describe difference compared with time point zero.

serine 473 by other kinases such as mTOR complex 2 (mTORC2) or DNA-dependent protein kinase (DNA-PK) $(19,20)$. The mechanisms involved in flow-induced phosphorylation and activation of AKT in endothelial cells are still unknown.

Protein kinases $\mathrm{N}(\mathrm{PKN})$ are serine/threonine kinases formerly known as protein kinase C-related kinases (PRKs), which consist of the 3 isoforms PKN1, PKN2, and PKN3 (21, 22). PKN2 shares with the other isoforms an N-terminal antiparallel coiledcoil structure (HR1 domain) that binds to and is activated by Rho family GTPases (23-25) and a C-terminal kinase domain that can be phosphorylated by PDK1, thereby promoting PKN activation $(26,27)$. In vitro studies in various cell types have involved PKN2 in the regulation of different cellular functions including cytoskeletal organization, cell migration, as well as cell differentiation (28-32). Loss of PKN2 in mice results in morphogenetic and cardiovascular defects and is lethal at $\mathrm{E} 10.5(33,34)$.

Here, we show that PKN2 plays a central role in flow-induced eNOS regulation by mediating phosphorylation of eNOS at serine 1177 via AKT and by direct phosphorylation of eNOS at serine 1179.

\section{Results}

Flow induces phosphorylation of human eNOS at serines 1177 and 1179. In a mass spectrometry-based phosphoproteomic analysis of bovine aortic endothelial cells (BAECs), we found that eNOS was phosphorylated in response to laminar flow not only at serines 635 and 1179, which correspond to human eNOS serines 633 and 1177, but also at serine 1181, which corresponds to human eNOS serine 1179 (Figure 1, A and B). Increased phosphorylation of bovine and human eNOS at serines 1181 and 1179, respectively, could be validated in BAECs and human umbilical arterial endothelial cells (HUAECs) using a phosphorylation site-specific antibody (Figure 1, C and D). This antibody specif- ically recognized a phosphomimetic mutant of human eNOS, in which serine 1179 was replaced by aspartic acid (S1179D), while a phosphomimetic mutant of eNOS at serine 1177 (S1177D) was specifically recognized by the anti-phosphoserine 1177 antibody (Figure 1E). Serine residue 1179 of human eNOS and the surrounding sequence were highly conserved in other species (Figure 1A). Expression of the S1179D mutant of human eNOS resulted in increased NO formation, as was seen with the S1177D mutant, when expressed in endothelial cells after knockdown of endogenous eNOS (Figure 1F and Supplemental Figure 1; supplemental material available online with this article; https:/doi. org/10.1172/JCI145734DS1). A phosphomimetic serine 1177 and 1179 double-mutant (S1177D/S1179D) induced a significantly greater increase in NO formation than did either mutant alone (Figure 1F). This additive effect indicates that both phosphorylation events acted independently of each other. After knockdown of endogenous eNOS in HUAECs, reexpression of human eNOS mutants, which cannot be further phosphorylated (S1177A and S1179A) only partially rescued flow-induced NO formation compared with cells in which WT eNOS was reexpressed, and a S1177A/S1179A double-mutant showed an even more reduced ability to rescue flow-induced NO formation (Figure $1 \mathrm{G}$ ). Similarly, carotid arteries from eNOS ${ }^{-/-}$mice, in which the S1177A and S1179A mutants of eNOS were reexpressed by adeno-associated virus 2-QuadYF-mediated (AAV2-QuadYF-mediated) transduction, only partially rescued flow-induced vasorelaxation compared with vessels that expressed WT eNOS (Figure $1 \mathrm{H})$. This indicates that, in addition to phosphorylation at serine 1177, human eNOS was also phosphorylated at serine 1179 in response to flow and that serine 1179 phosphorylation contributed to the flow-induced increase in eNOS activity. Flow-induced eNOS phosphorylation at serine 1179 was not affected by knockdown of AKT1, the major isoform of AKT expressed in endothelial cells, whereas AKT1 knockdown strongly inhibited flow-induced eNOS phosphorylation at serine 1177 (Figure 1I). Thus, flow-induced phosphorylation of human eNOS at serine 1179 occurred in an AKT-independent manner.

PKN2 is activated by flow and mediates the phosphorylation of eNOS at serines 1177 and 1179. In an attempt to identify protein kinases involved in flow-induced eNOS phosphorylation at serine 1179, we performed an siRNA-mediated knockdown of 25 protein kinases highly expressed in HUAECs and determined the effect on flow-induced phosphorylation of eNOS at serines 1177 and 1179 (Figure 2A and Supplemental Figure 2A). Among the kinases whose knockdown significantly reduced flow-induced eNOS phosphorylation at serine 1179 was PKN2 (Figure 2A). An alternative siRNA directed against PKN2 blocked flow-induced eNOS phosphorylation at serine 1179 and at serine 1177 (Figure 2B) as well as flow-induced increases in NO levels in cellular supernatants as determined by the measurement of nitrite/nitrate (NOx) levels in HUAECs (Figure 2C). We observed corresponding effects after PKN2 knockdown in BAECs (Figure 2, D and E). We found that PKN2 was the major PKN isoform expressed in different endothelial cells, followed by PKN1, whereas PKN3 showed lower endothelial expression (Supplemental Figure 3A). Knockdown of PKN1 expression had no effect on flow-induced eNOS phosphorylation or NO formation in HUAECs (Supplemental Figure 3, C and D). 
A

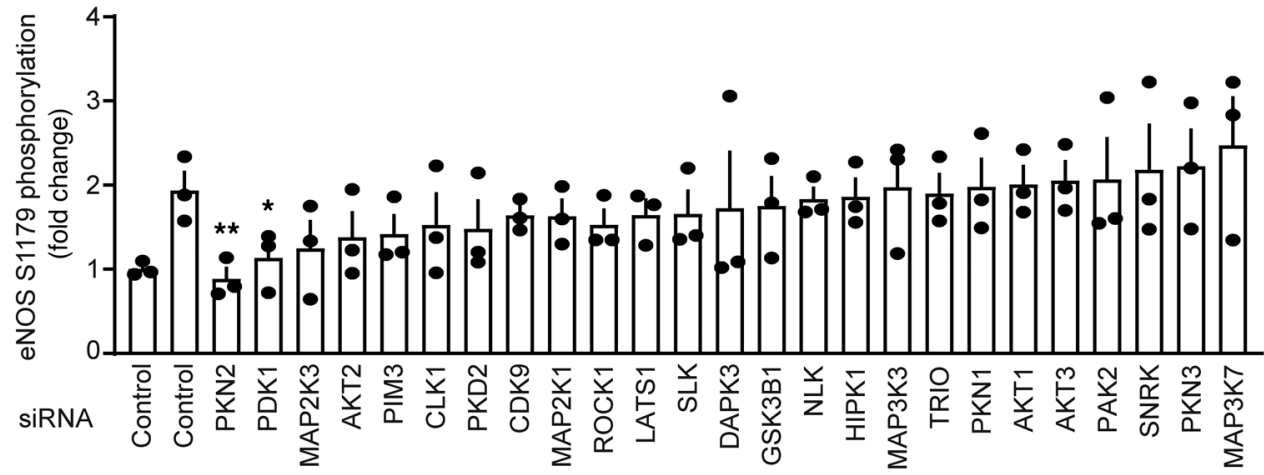

Static

Laminar flow

B

HUAECs

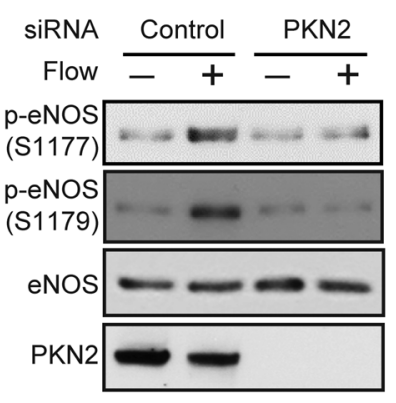

D

BAECs

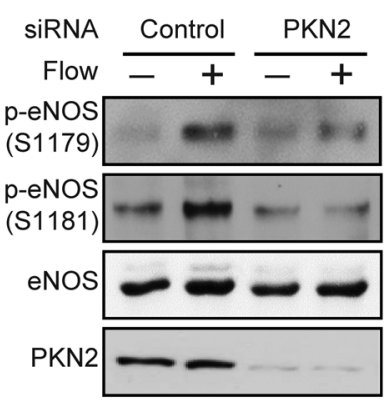

F IP Anti-PKN2 IgG $\begin{array}{llllll}\text { Flow (min) } & 0 & 5 & 15 & 30 & 30\end{array}$
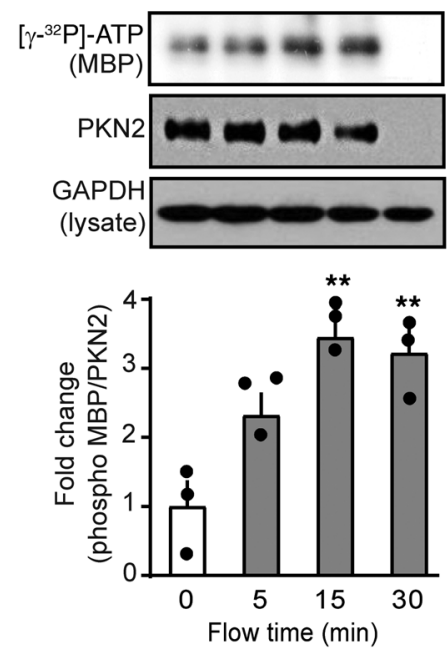
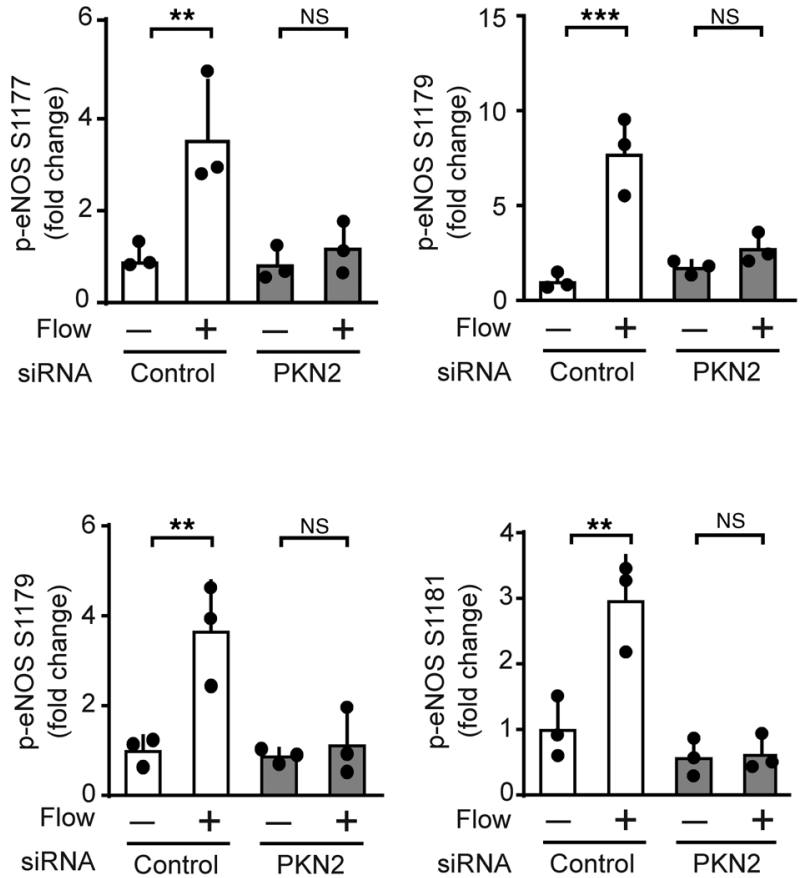

G

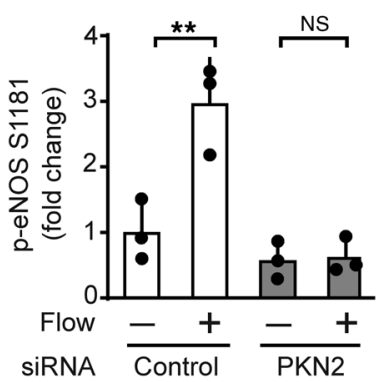

H
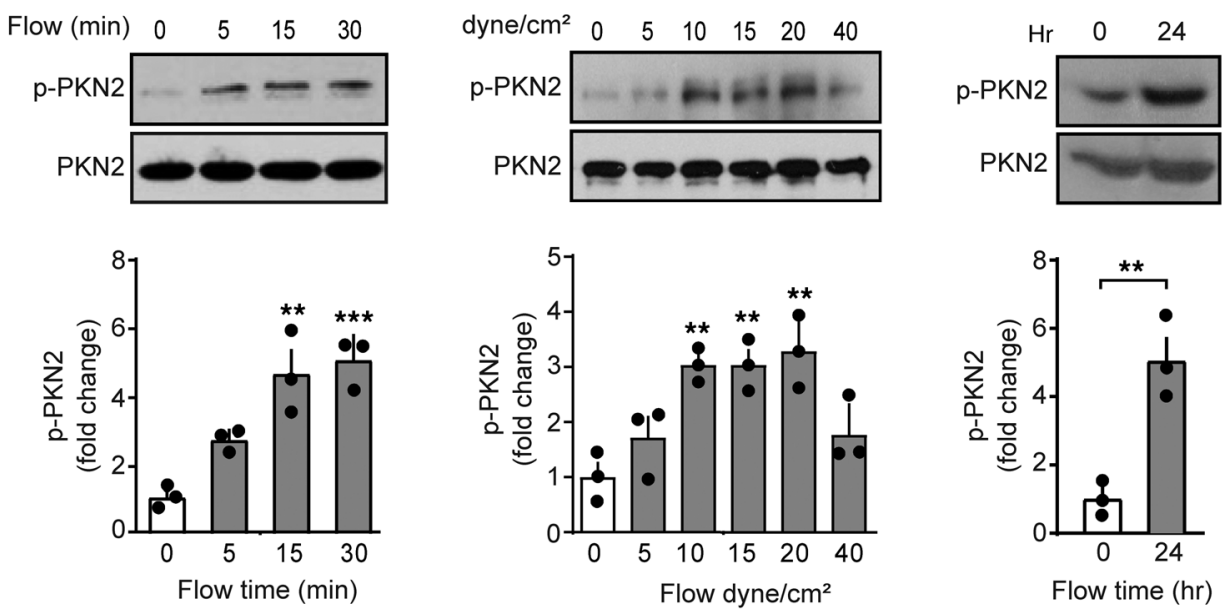

C

HUAECS

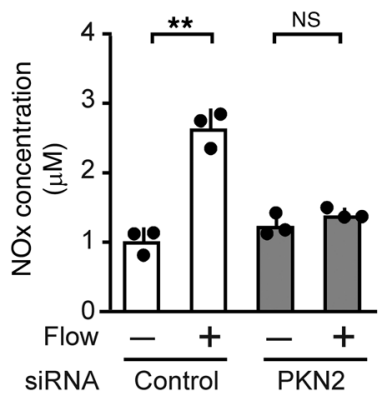

E

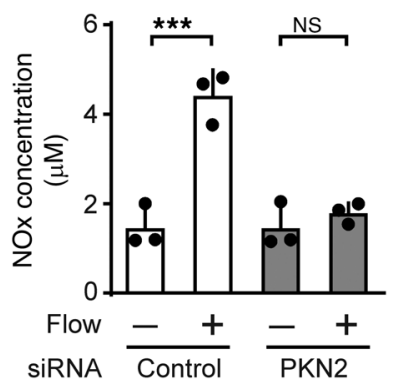

I

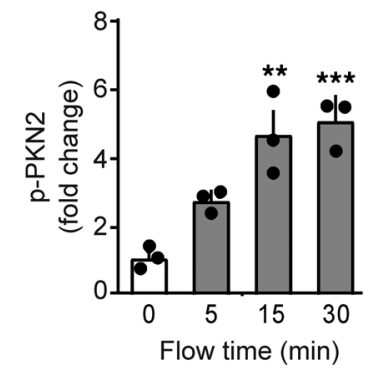

Flow time (hr) 
Figure 2. PKN2 is activated by flow and mediates the phosphorylation of eNOS at serines 1177 and 1179. (A) HUAECs were transfected with a contro SiRNA or siRNAs directed against the indicated protein kinases and were kept under static conditions or were exposed to laminar flow (15 dynes/ $\mathrm{cm}^{2}$ ) for 15 minutes. Shown is the ratio of flow-induced phosphorylation of eNOS at serine 1179 in cells transfected with a control siRNA and an siRNA against a particular protein kinase. The plot shows the ranked average ratios of 3 independent experiments. (B-E) HUAECs (B and $\mathbf{C}$ ) and BAECs (D and $\mathbf{E}$ ) were transfected with control or an siRNA against PKN2 and were exposed to laminar flow ( $\left.15 \mathrm{dynes} / \mathrm{cm}^{2}\right)$. Total and p-eNOS as well as PKN2 levels were determined by immunoblotting (B and $\mathbf{D}$ ), and NOx levels were determined in the supernatants ( $\mathbf{C}$ and $\mathbf{E}$ ). Graphs show the densitometric evaluation of the blots ( $n=3$ independent experiments). (F-I) HUAECs were exposed to laminar flow ( 15 dynes $/ \mathrm{cm}^{2}$, if not stated otherwise) for the indicated durations. Thereafter, PKN2 was immunoprecipitated, and its kinase activity was determined as described in Methods $(\mathbf{F})$, and total as well as p-PKN2 levels were determined by immunoblotting (G-I). Graphs show the densitometric evaluation of the blots $(n=3$ independent experiments). Data represent the mean $\pm \mathrm{SEM}$. ${ }^{*} P \leq 0.05$, ${ }^{* *} P \leq 0.01$, and ${ }^{* *} P \leq 0.001$, by 2 -way ANOVA with Bonferroni's post hoc test (A, compared with control, laminar flow), 1-way ANOVA, with Tukey's post hoc test (B-H), and unpaired, 2-tailed Student's $t$ test (I).

To test whether PKN2 is activated by laminar flow in endothelial cells, we determined the effect of laminar shear stress on PKN2 kinase activity. Laminar flow increased PKN2 kinase activity, as shown by an in vitro kinase assay on immunoprecipitated PKN2 using myelin basic protein (MBP) as a substrate (Figure 2F). Within 5 minutes of applying laminar flow to endothelial cells, we detected phosphorylation of PKN2 at threonine 816, which has been shown to result in PKN2 activation (27) (Figure 2G). Phosphorylation of PKN2 required high shear rates of at least 10 dynes/ $\mathrm{cm}^{2}$ and lasted for at least 24 hours (Figure 2, $\mathrm{H}$ and I).

Flow-induced PKN2 activation involves Piezo1, $G_{q} / G_{11}$, and PDK1, but not PI3K. RhoA, a known regulator of PKN2, was not involved in flow-induced PKN2 activation, as knockdown of RhoA did not affect flow-induced PKN2 phosphorylation (Supplemental Figure 4A). Since PDK1 has been shown to phosphorylate and thereby activate PKN2 $(26,27,35)$, and since knockdown of PDK1 inhibited flow-induced phosphorylation of human eNOS at serine 1179 to a degree comparable to that seen with knockdown of PKN2 (Figure 2A), we tested its involvement in flow-induced PKN2 activation. Knockdown of PDK1, in fact, blocked flow-induced PKN2 phosphorylation as well as phosphorylation of eNOS at serines 1177 and 1179 (Figure 3A). The PDK1 activator PS48 induced a strong phosphorylation of PKN2 as well as of serines 1177 and 1179 of eNOS (Figure 3, B and C), and knockdown of PKN2 inhibited PS48-induced eNOS phosphorylation at serines 1177 and 1179 (Figure 3C). Similarly, NO formation induced by PS48 was also strongly inhibited by knockdown of PKN2 (Figure 3D). This indicates that flow-induced PKN2 activation resulting in eNOS phosphorylation was mediated by PDK1.

To understand the upstream regulation of PDK1 and PKN2 in flow-induced signaling, we tested the effect of knockdown or inhibition of known upstream mediators of flow-induced eNOS activation on flow-induced phosphorylation of PKN2. Knockdown of Piezo1 inhibited phosphorylation of both PDK1 and PKN2 as well as eNOS phosphorylation at serines 1177 and 1179 (Figure 3E), and the Piezo1 activator Yoda1 induced phosphorylation of PDK1 and
PKN2 (Figure 3F). The effect of Piezo1 activation on PKN2 phosphorylation was blocked after suppression of PDK1 expression (Figure 3G). Flow-induced activation of Piezo1 resulting in eNOS activation has been shown to involve the release of ATP, activation of the endothelial $P 2 Y_{2}$ receptor, and subsequent $G_{q} / G_{11}$-mediated signaling $(15,16)$. Knockdown of $\mathrm{P}_{2} \mathrm{Y}_{2}$ expression with a specific siRNA or pharmacological inhibition of $G_{q} / G_{11}$ by $Y M-254890$ (36) inhibited flow-induced phosphorylation of both PDK1 and PKN2 (Figure 3, H and I). Thus, flow-induced activation of PDK1 and PKN2 involves Piezo1 and $P 2 Y_{2} / G_{q} / G_{11}$-mediated signaling.

Piezo1 and $G_{q} / G_{11}$-mediated signaling has been shown to mediate flow-induced activation of the mechanosignaling complex consisting of PECAM-1, VE-cadherin, and VEGFR2 as well as PI3K and AKT $(15,16)$. We therefore tested the effect of inhibition of VEGFR2 by Ki8751 and of PI3K by wortmannin or LY294002 as well as the effect of an AKT1 knockdown on flow-induced phosphorylation of PDK1, PKN2, and eNOS. Inhibition of VEGFR2 and PI3K had no effect on flow-induced PDK1 or PKN2 phosphorylation, whereas it blocked the flow-induced phosphorylation of eNOS at serine 1177 but not the phosphorylation of eNOS at serine 1179 (Figure 4A). As seen with inhibition of VEGFR2 and PI3K, knockdown of AKT1 did not affect PKN2 phosphorylation in response to flow (Figure $4 \mathrm{~B}$ ). This observation indicated that the mechanosignaling complex and PI3K/AKT were not involved in flow-induced activation of PDK1 and PKN2 downstream of Piezo1 and $G_{\mathrm{q}} / \mathrm{G}_{11}$.

We then tested whether the increase in intracellular free $\mathrm{Ca}^{2+}$ $\left(\left[\mathrm{Ca}^{2+}\right]_{\mathrm{i}}\right)$ induced by flow through Piezo1 and $\mathrm{G}_{\mathrm{q}} / \mathrm{G}_{11}$-mediated signaling (16) is involved in flow-induced activation of PDK1 and PKN2. In fact, chelation of $\left[\mathrm{Ca}^{2+}\right]_{\mathrm{i}}$ by BAPTA-AM [1,2-Bis(2-aminophenoxy)ethane- $N, N, N^{\prime}, N^{\prime}$-tetraacetic acid tetrakis(acetoxymethyl ester)] blocked the flow-induced phosphorylation of PDK1 and PKN2 (Figure 4C), and, in addition to the Piezo1 activator Yoda1, the $\mathrm{Ca}^{2+}$ ionophore ionomycin also induced PDK1 and PKN2 phosphorylation (Figure 4D). Phosphorylation of PKN2 in response to ionomycin was blocked after suppression of PDK1 expression (Figure 4E). These data indicate that flow-induced PKN2 phosphorylation was mediated by PDK1 in a manner dependent on Piezo1, ATP/P2Y, $\mathrm{G}_{\mathrm{q}} / \mathrm{G}_{11}$, and $\mathrm{Ca}^{2+}$, but independent of PI3K (Figure 4F).

PKN2 mediates eNOS activation by AKT-dependent phosphorylation at serine 1177 and direct phosphorylation at serine 1179. Although activation of PDK1 and PKN2 appeared to occur independently of flow-induced activation of the mechanosignaling complex and PI3K, which has been shown to be required for flow-induced phosphorylation of eNOS at serine 1177 by AKT $(16,37)$, knockdown of PKN2 and PDK1 blocked flow-induced eNOS phosphorylation at serine 1177 (Figure 2B and Figure 3A). We therefore tested whether AKT activation by flow requires PKN2 and PDK1. As shown in Figure 5, A and B, knockdown of PKN2 and PDK1 had no effect on flow-induced AKT phosphorylation at serine 473 but strongly inhibited AKT phosphorylation at threonine 308. Flow-induced AKT phosphorylation at serine 473 was instead blocked by knockdown of the mTORC2 component Rictor (Figure 5C). Thus, PDK1 and PKN2 acted upstream of AKT by mediating phosphorylation at threonine 308 but not at serine 473 , which was phosphorylat- 
A

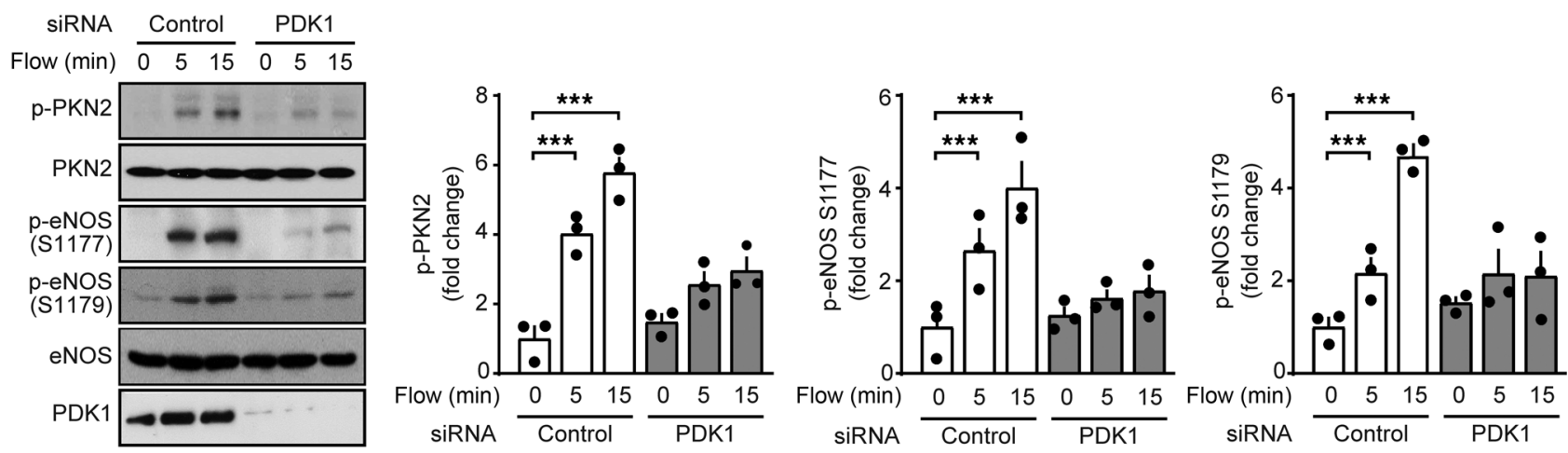

B

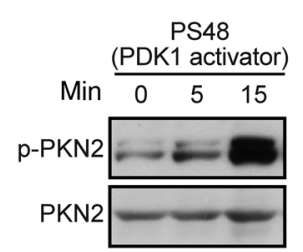

E

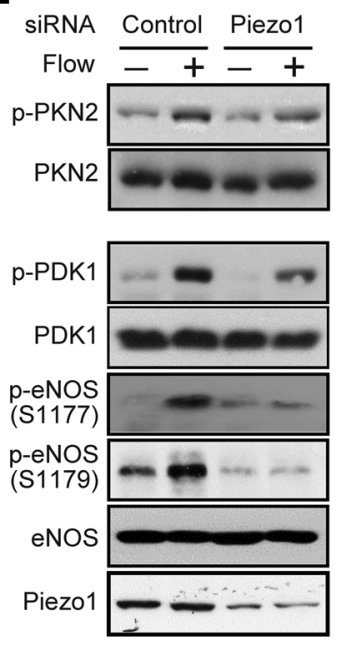

C

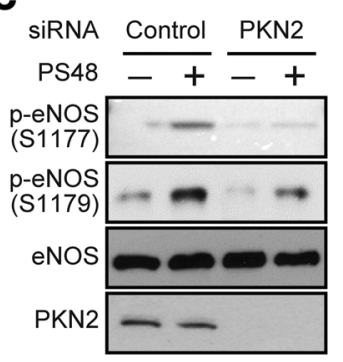

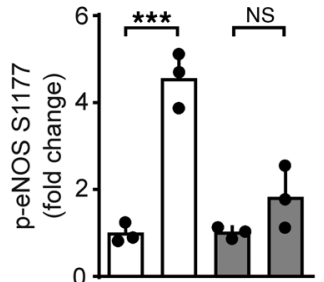

PS48 -+++ siRnA $\overline{\text { Control }} \overline{\text { PKN2 }}$

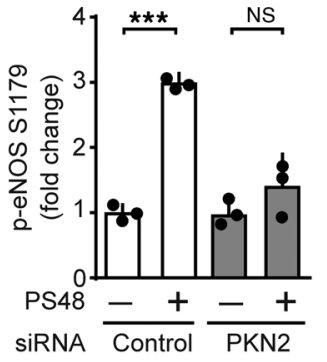

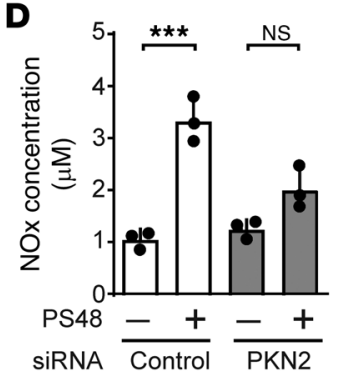
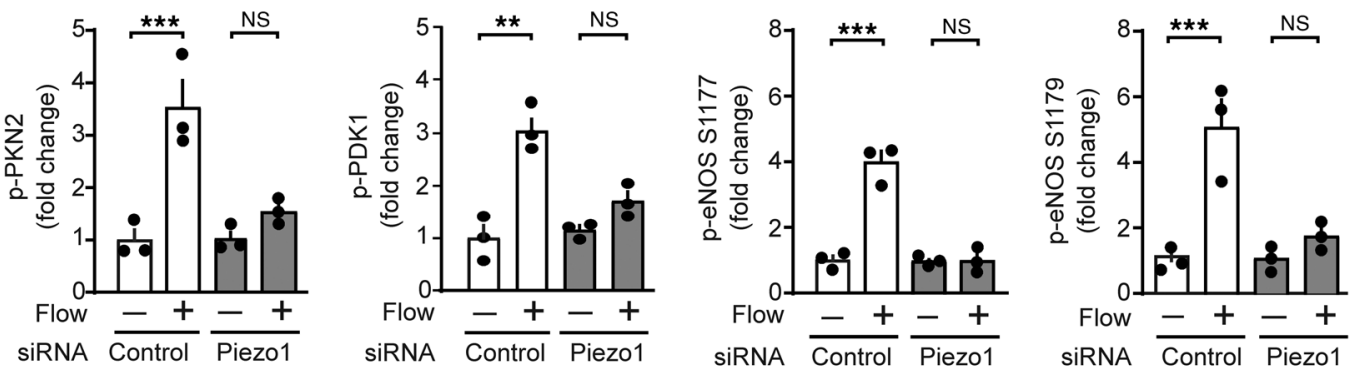

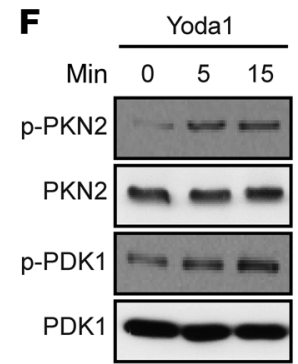

G
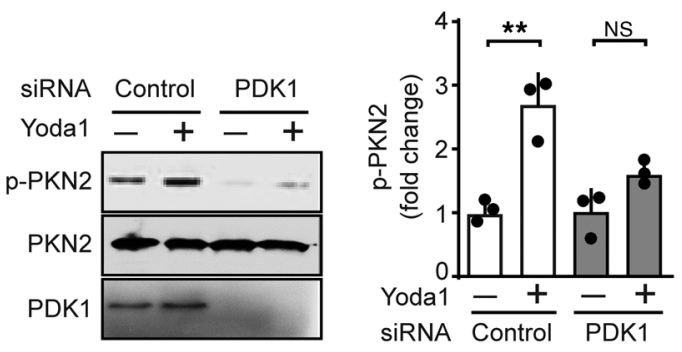

H

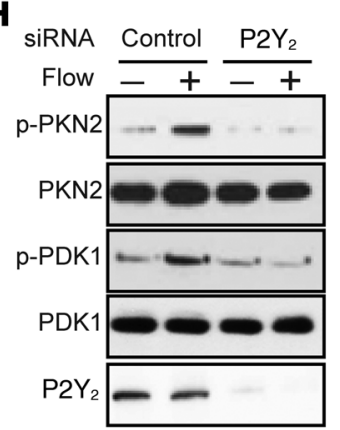

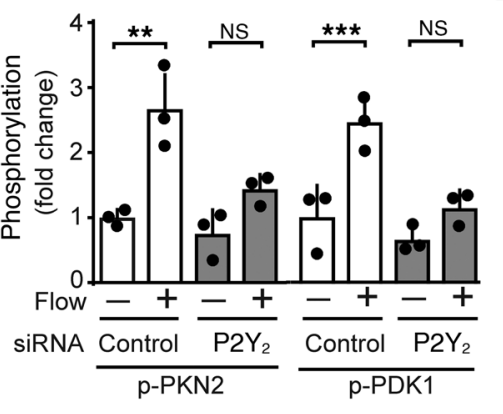

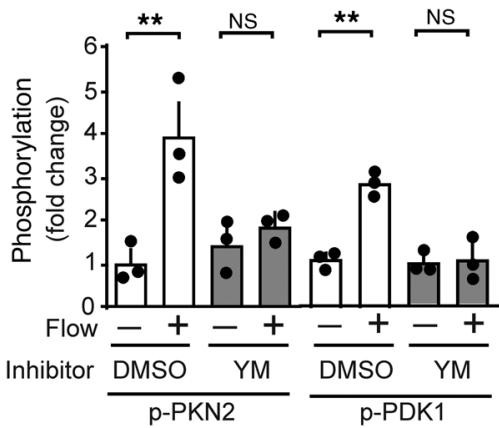


Figure 3. Flow-induced PKN2 activation involves Piezo1, $\mathrm{G}_{q} / \mathrm{G}_{11}$, and PDK1. (A-I) HUAECs were transfected with a control siRNA or siRNAs directed against PDK1 (A and $\mathbf{G})$, PKN2 (C and D), Piezo1 (E), or P2Y 2 (H), or were left untransfected (B and $\mathbf{F}$ ) or were pretreated or not with 100 nM YM-254890 (YM) (I) for 30 minutes. Thereafter, cells were exposed to laminar flow (15 dynes $/ \mathrm{cm}^{2}$ ) for 15 minutes for the indicated durations (A $\mathbf{E}, \mathbf{H}$, and $\mathbf{I}$ ), or to $5 \mu \mathrm{M}$ PS48 for 15 minutes for the indicated durations (B-D), or to $1 \mu \mathrm{M}$ Yoda1 for 15 minutes for the indicated durations (F and C). Total and phosphorylated levels of the indicated proteins in lysates were determined by immunoblotting (A-C, E-I), and the NOx concentration was measured in the supernatant (D). Graphs in A, C, and $\mathbf{G}-\mathbf{I}$ show densitometric analyses of the blots ( $n=3$ independent experiments). Data represent the mean $\pm \mathrm{SEM}$. ${ }^{* *} P \leq 0.01$ and ${ }^{* * *} P \leq 0.001$, by 1 -way ANOVA with Tukey's post hoc test.

ed via mTORC2. Consistent with this, the PDK1 activator PS48 induced AKT phosphorylation at threonine 308 but not at serine 473, and this effect was blocked after knockdown of PKN2 (Figure 5D). In addition, expression of a constitutively active mutant of PKN2 induced phosphorylation of eNOS at both serine 1177 and serine 1179, however, only phosphorylation at serine 1177 was strongly inhibited after knockdown of AKT1 (Figure 5E). Similarly, the increased NO production induced by the expression of constitutively active PKN2 was partially reduced by knockdown of AKT1 as well as by overexpression of the AKT1 phosphosite mutant T308A (Figure 5, F and G). These data indicate that PKN2 was involved in flow-induced AKT phosphorylation at threonine 308 and that AKT mediated the PKN2-induced eNOS activation at serine 1177.

As expected from the initial observation that flow-induced phosphorylation of human eNOS at serine 1179 did not involve AKT (Figure 1H), AKT1 knockdown had no effect on eNOS phosphorylation at serine 1179 induced by active PKN2, but inhibited PKN2-induced eNOS phosphorylation at serine 1177 (Figure 5E). To determine whether PKN2 can directly phosphorylate eNOS at serine 1179, we first tested whether PKN2 and eNOS physically interact. When PKN2 was precipitated from endothelial cells under static conditions, no signal could be detected with an antieNOS antibody in the precipitate. However, after 15 minutes of laminar flow, a relatively strong eNOS signal was seen in the PKN2 precipitate (Figure 6A). When recombinant eNOS was incubated together with purified PKN2 in the presence of ATP, we observed strong phosphorylation of serine 1179, but not serine 1177 (Figure $6, \mathrm{~B}$ and C). These data indicate that PKN2 can interact with eNOS and phosphorylate serine 1179 of eNOS, suggesting that PKN2 induces eNOS activation in response to flow through AKT-dependent phosphorylation at serine 1177 as well as by direct phosphorylation of eNOS at serine 1179 (Figure 6D).

Endothelial PKN2 deficiency results in hypertension and loss of flow-induced vasodilation. To test whether PKN2-mediated endothelial eNOS activation is physiologically relevant, we generated mice with tamoxifen-inducible, endothelium-specific PKN2 deficiency (Tek-CreER $R^{T 2} P k n 2^{f / / l}$ mice, herein referred to as EC-Pkn2-KO mice) (Figure 7A). Precontracted aortic segments from induced EC-Pkn2-KO mice showed normal relaxation in response to acetylcholine (Figure 7B). However, endothelial loss of PKN2 resulted in a strong reduction of flow-induced dilation of precontracted mesenteric vessels (Figure 7C). We then test- ed the effect of an acute induction of endothelial PKN2 deficiency on telemetrically recorded blood pressure in conscious mice. Treatment of WT mice with tamoxifen resulted in a transient increase in arterial blood pressure (Figure 7D). However, induction of endothelial PKN2 deficiency led to a sustained 20 $\mathrm{mmHg}$ increase in arterial blood pressure. We detected a very similar increase in blood pressure after tamoxifen treatment of Cdh5-CreERT2 Pkn $2^{f / f l}$ mice (Supplemental Figure 5, A and B). Induction of endothelial Pkn1 deficiency had no effect on blood pressure (Figure 7D), consistent with a lack of any effect of Pkn1 knockdown on flow-induced eNOS activation in vitro. In parallel to the increase in arterial blood pressure after induction of endothelium-specific PKN2 deficiency, we observed a reduced phosphorylation of murine mesenteric artery eNOS at serines 1176 and 1178, corresponding to serines 1177 and 1179 in human eNOS, respectively (Figure 7E). The reduced phosphorylation of eNOS in mice with induced endothelial PKN2 deficiency was accompanied by a decrease in plasma NOx levels (Figure 7F). These data indicate that the acute loss of PKN2-mediated signaling in the endothelium results in reduced eNOS activity, leading to increased vascular tone and blood pressure.

\section{Discussion}

The regulation of eNOS is a complex process involving different upstream stimuli and several mechanisms including posttranslational modifications $(3,4,9)$. Fluid shear stress exerted by the flowing blood is one of the most important stimuli of eNOS activity, which is centrally involved in basal control of vascular functions such as vascular tone and blood pressure. In the past, 2 major pathways were described to regulate eNOS activity in response to fluid shear stress. One is initiated by flow-induced release of adrenomedullin and subsequent activation of cAMP formation and protein kinase $A$ through the adrenomedullin receptor resulting in the phosphorylation of human eNOS at serine $633(4,8,9,12,38)$. The other one is initiated by the release of ATP, which, through activation of P2Y receptors and the $G_{q} / G_{11}$-mediated signaling pathway, results in activation of AKT and phosphorylation of human eNOS at serine $1177(3,4,6,7,16)$. Upstream regulation of both pathways has been shown to involve activation of the mechanosensitive cation channel Piezo1 $(12,15)$. Although previous research had shown that flow-induced $G_{\mathrm{q}} / \mathrm{G}_{11}$-mediated activation of AKT involves a mechanosignaling complex including VEGFR2 (16, 37), the direct mechanism of flow-induced AKT activation has remained unclear. Here, we show that AKT activation and subsequent eNOS phosphorylation at serine 1177 in response to flow required PKN2 and that PKN2 in addition also led to the phosphorylation of eNOS at serine 1179. We also demonstrate that these effects were additive, and we identified the upstream regulation of flow-induced PKN2 activation, which involved P2 $Y_{2}$ - and $G_{q} / G_{11}$-mediated increases in $\left[\mathrm{Ca}^{2+}\right]_{i}$ and subsequent PDK1 activation (Figure 6D). The fact that acute loss of endothelial PKN2 led to reduced eNOS activity, reduced flow-induced vasodilation, and hypertension in vivo indicates that this new PKN2-mediated signaling pathway in endothelial cells is constantly activated to mediate fluid shear stress-induced endothelial NO formation and vasodilation. 
A
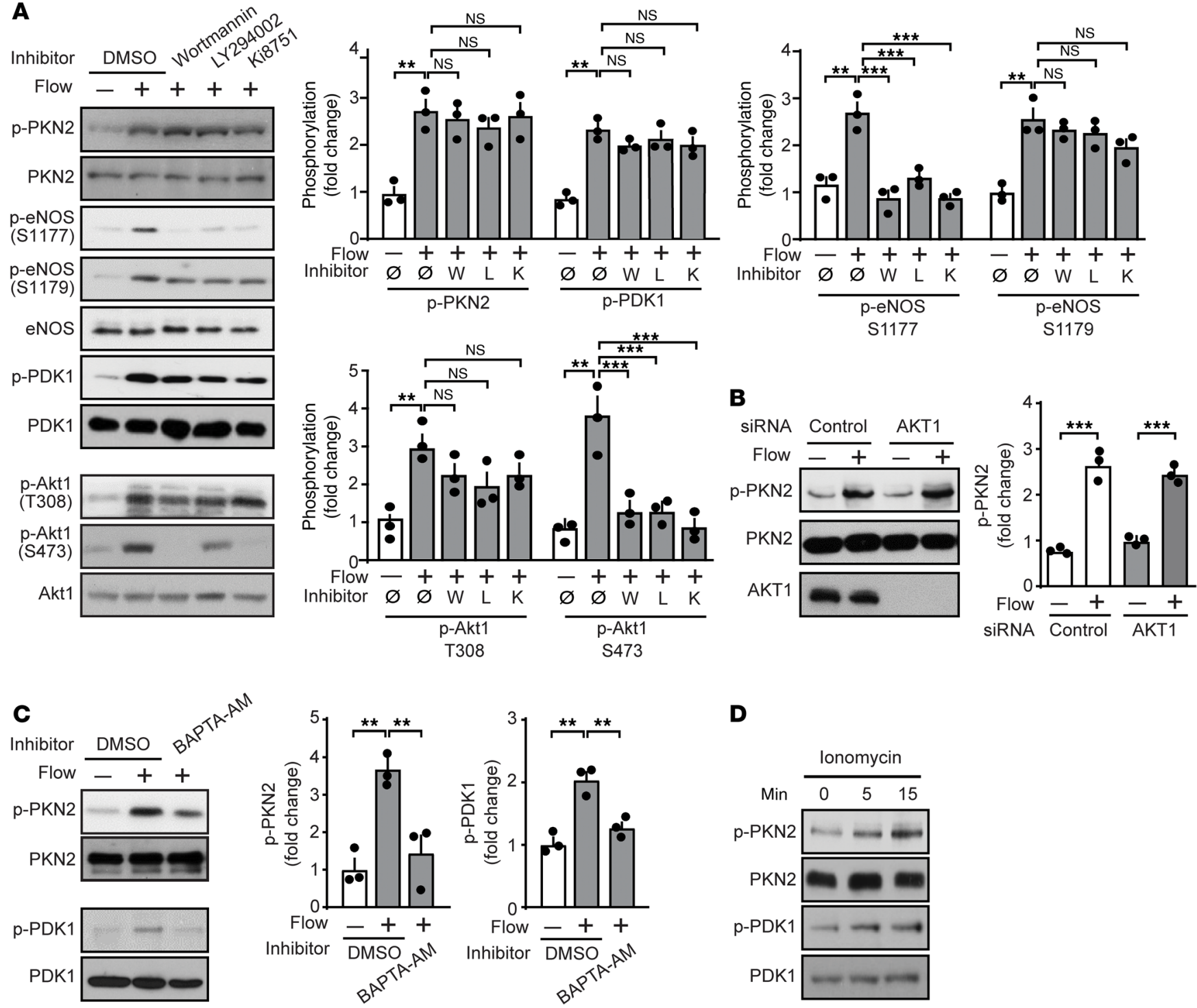

E
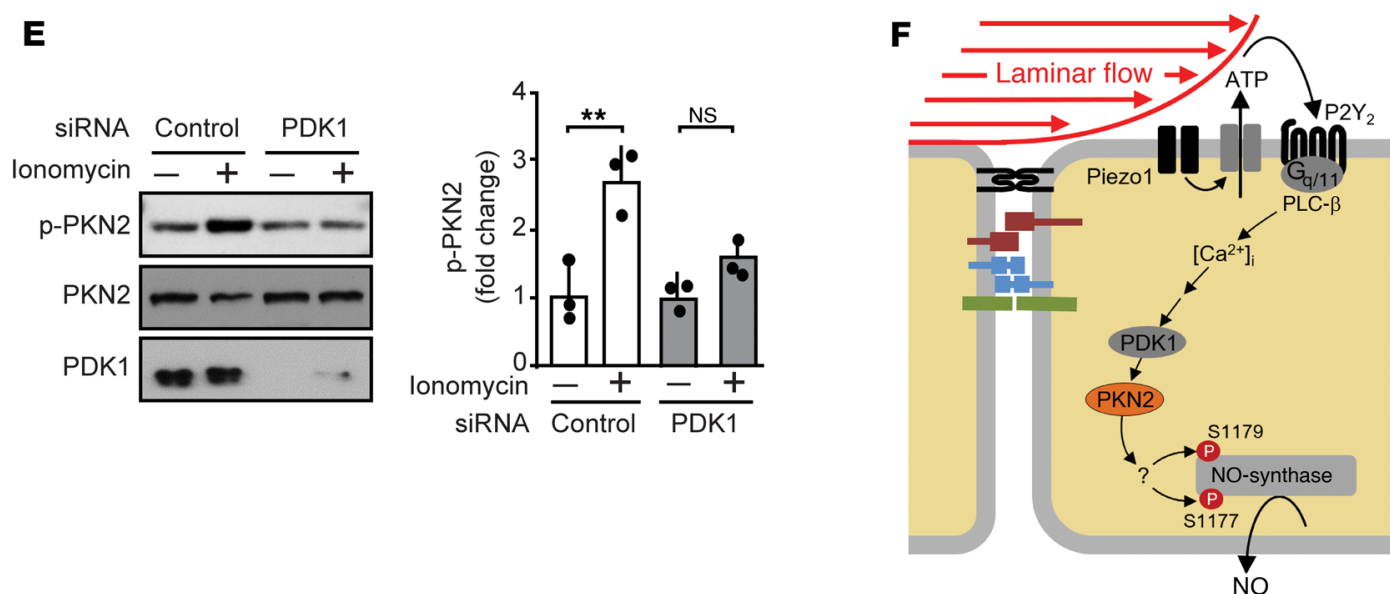

Figure 4. Flow-induced PKN2 activation involves $\mathrm{Ca}^{2+}$ but not PI3K and AKT. (A-E) HUAECs were preincubated for 30 minutes with the PI3K inhibitor wortmannin $(100 \mathrm{nM})$ or LY294002 $(10 \mu \mathrm{M})$ or the VEGFR2 inhibitor Ki8751 (500 nM) (A); or with $20 \mu \mathrm{M}$ BAPTA-AM (C); or were transfected with a control siRNA or siRNAs

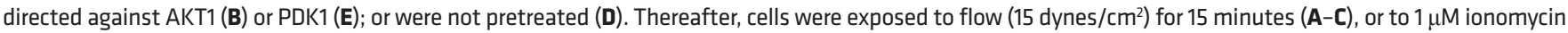
( $\mathbf{D}$ and $\mathbf{E}$ ) for 15 minutes or the indicated durations. Total and phosphorylated protein levels in lysates were determined by immunoblotting. Graphs show the densitometric analysis of the blots ( $n=3$ independent experiments). The control immunoblot for AKT1 in $\mathbf{B}$ is from the same experiment as the one shown in Figure 11. (F) Model of the mechanism mediating laminar flow-induced PKN2 activation resulting in eNOS phosphorylation and activation. Data represent the mean \pm SEM. ${ }^{* *} P \leq 0.01$ and ${ }^{* *} P \leq 0.001$, by 1-way ANOVA, with Tukey's post hoc test. 
The central role of AKT in eNOS phosphorylation and activation is well established $(6,7,39)$, and viral transduction of a constitutively active AKT resulted in increased NO release and enhanced blood flow in vivo (40). Similar results were observed after endothelial cell-specific expression of an active version of AKT, which resulted in increased NO production (41). That AKT-mediated regulation of eNOS activity in vivo is mediated by phosphorylation of eNOS at serine 1177 is further supported by the previous observation that the corresponding phosphomimetic murine eNOS mutant S1176D can rescue the vascular phenotype observed in AKT1-deficient mice (39), as well as by the finding that endothelial cell-specific AKT1-deficient mice have decreased plasma NO levels, reduced phosphorylation of murine eNOS at serine 1176 in blood vessels, and increased blood pressure (18). The mechanism by which AKT is activated by fluid shear stress has, however, remained unclear. A general requirement for AKT activation is the stimulation of $\mathrm{PI} 3 \mathrm{~K}$, leading to the formation of $\mathrm{PIP}_{3}$, which recruits AKT to the membrane through interaction with its PH domain $(19,20,42)$. Consistent with this, fluid shear stress has been shown to induce activation of PI3K $(37,43)$. However, full activation of the AKT kinase activity requires phosphorylation of AKT at threonine 308 in the catalytic domain of the kinase, which typically involves PDK1 (19, $20,44,45)$ as well as phosphorylation of serine 473 in a C-terminal hydrophobic motif mainly mediated by mTORC2 $(46,47)$. Whether flow-induced endothelial AKT activation also involves PDK1- and mTORC2-mediated phosphorylation was, however, unknown. Our data clearly show that disruption of mTORC2 by suppression of Rictor expression blocked flow-induced AKT phosphorylation at serine 473 , but not at threonine 308 . Flow-induced AKT phosphorylation at threonine 308 was blocked after knockdown of both PDK1 and PKN2, whereas, in both cases, phosphorylation at serine 473 was unaffected. The specific phosphorylation of AKT at threonine 308 could be mimicked by the PDK1 activator PS48, which also induced a strong phosphorylation of PKN2. Interestingly, the effect of PS48 on AKT phosphorylation at threonine 308 was blocked after knockdown of PKN2. This indicated that flow-induced AKT phosphorylation at threonine 308 through PDK1 did not occur directly, but rather was mediated by PKN2 in endothelial cells exposed to flow. This function of PKN2 as a mediator of PDK1-induced phosphorylation of AKT at threonine 308 is consistent with several in vitro reports showing that PKN2 is a substrate of PDK1 and can be activated through PDK1-mediated phosphorylation $(26,27,35)$. In addition, there is also evidence that PKN2 and AKT1 interact $(48,49)$ and that PKN2 can activate AKT $(32)$. We conclude that flow-induced activation of endothelial AKT occurred via PI3K/ mTORC2-mediated phosphorylation at serine 473 and phosphorylation at threonine 308 through an atypical mechanism involving PKN2, which is activated by PDK1 (Figure 6D).

PKNs have been described to be activated by binding to members of the Rho family of GTPases through a Rho-binding motif known as the HR1 domain (23-25). However, we found no evidence for a role of Rho-GTPases in flow-induced endothelial PKN2 activation. This is consistent with published data that RhoA is not activated in response to acute flow in endothelial cells (50). Rather, we found that flow-induced PKN2 activation in endothelial cells was mediated by PDK1. Interestingly, this PDK1-mediated PKN2 activation was independent of PI3K and VEGFR2, as their inhibition did not affect flow-induced phosphorylation of PKN2 and PDK1, whereas inhibition of PI3K and VEGFR2 blocked flow-induced AKT phosphorylation at serine $473(16,51)$. This indicates that flow-induced activation of PDK1/ PKN2 occurred independently of the mechanosignaling complex consisting of PECAM-1, VE-cadherin, and VEGFR2, which mediates flow-induced activation of PI3K (37). Instead, we found that flow-induced activation of PKN2 was blocked after knockdown of Piezo1 and P2Y $Y_{2}$, inhibition of $G_{q} / G_{11}$, and chelation of $\left[\mathrm{Ca}^{2+}\right]_{i}$ using BAPTA-AM, while it could be induced by the Piezo1 activator Yoda1 and the calcium ionophore ionomycin. This strongly indicates that flow-induced activation of PDK1 and PKN2 was a calcium-mediated process downstream of Piezo1 and $G_{q} / G_{11}$ (Figure 6D). This is consistent with earlier data showing that $\mathrm{Ca}^{2+}$ can activate PDK1 (52) and that $\mathrm{Ca}^{2+}$-induced phosphorylation of AKT at threonine 308 involves PDK1 (53).

Serine 1177 is one of the most extensively studied phosphorylation sites of human eNOS, and several in vitro studies have shown that a phosphomimetic mutant, in which serine 1177 is replaced by aspartic acid (S1177D), has an increased basal activity $(6,7)$. Conversely, endothelial cells expressing the phosphosite mutant S1177A show reduced flow-induced NO formation (12). Knockin mice expressing the corresponding phosphomimetic S1176D mutant of mouse eNOS show increased endothelium-dependent vasodilation, whereas mice expressing the phosphosite mutant S1176A have reduced endothelium-dependent vasodilation and increased blood pressure (54-56). Although this represents strong evidence for a role of serine 1177 phosphorylation in the regulation of human eNOS activity in vitro and in vivo, a recent in vitro study has challenged this view and found that eNOS phosphorylation at serine 1177 does not correlate with increased activity of the enzyme (57). The reason for this discrepancy is still unclear, but we did not analyze flow-induced effects in this study. Mechanistically, it has been suggested that the C-terminal region of the enzyme in which serine 1177 is located has an autoinhibitory function and that phosphorylation at serine 1177 reduces this autoinhibition (58-60). Our data show that, in addition to serine 1177, serine 1179 in human eNOS as well as the corresponding serine residue in bovine eNOS were phosphorylated in response to flow. This serine residue is highly conserved in different species, and its phosphorylation might have been overseen in previous analyses. We show that serines 1177 and 1179 were phosphorylated independently of each other by different mechanisms involving the classic PI3K/ mTORC2/AKT pathway as well as by a new mechanism mediated by PKN2. Importantly, both phosphorylation events promoted eNOS activation in an additive fashion. Whether the additivity of the effects of both phosphorylations on eNOS activity was due to the same or different mechanisms is currently not clear.

Our results identify serine 1179 of human eNOS as an additional phosphorylation site, that, together with the well-established serine 1177 phosphorylation site, additively regulated eNOS activity. Both serine residues were phosphorylated in response to fluid shear stress through different pathways - a PI3K-dependent pathway and an mTORC2-dependent pathway - resulting in AKT phosphorylation at serine 473 to induce serine 1177 phos- 
A

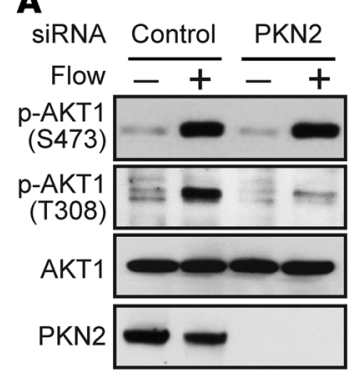

B

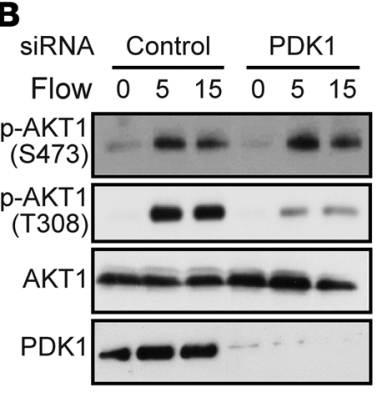

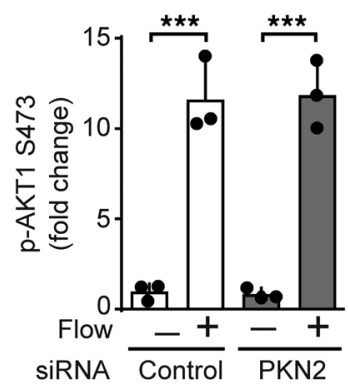

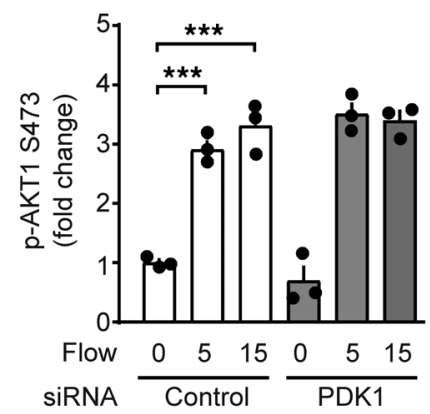

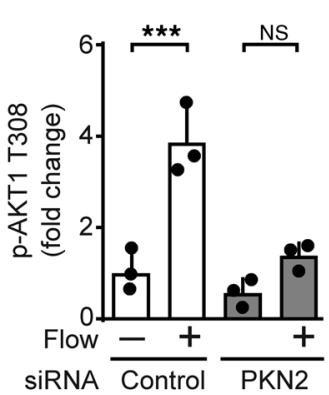

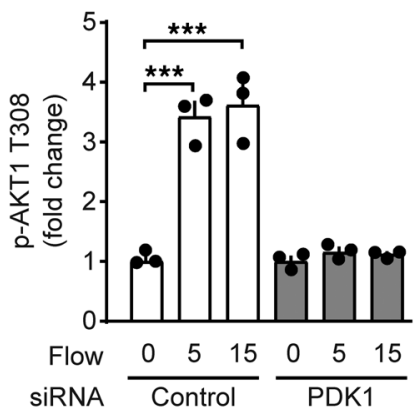

$\mathbf{F}$

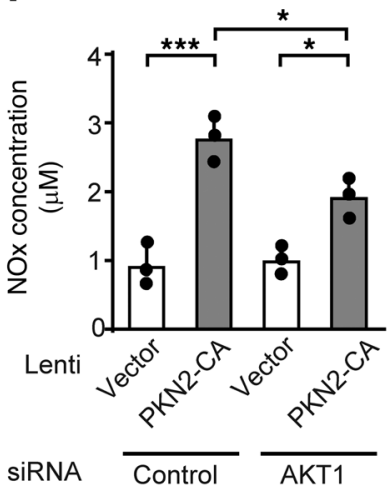

C
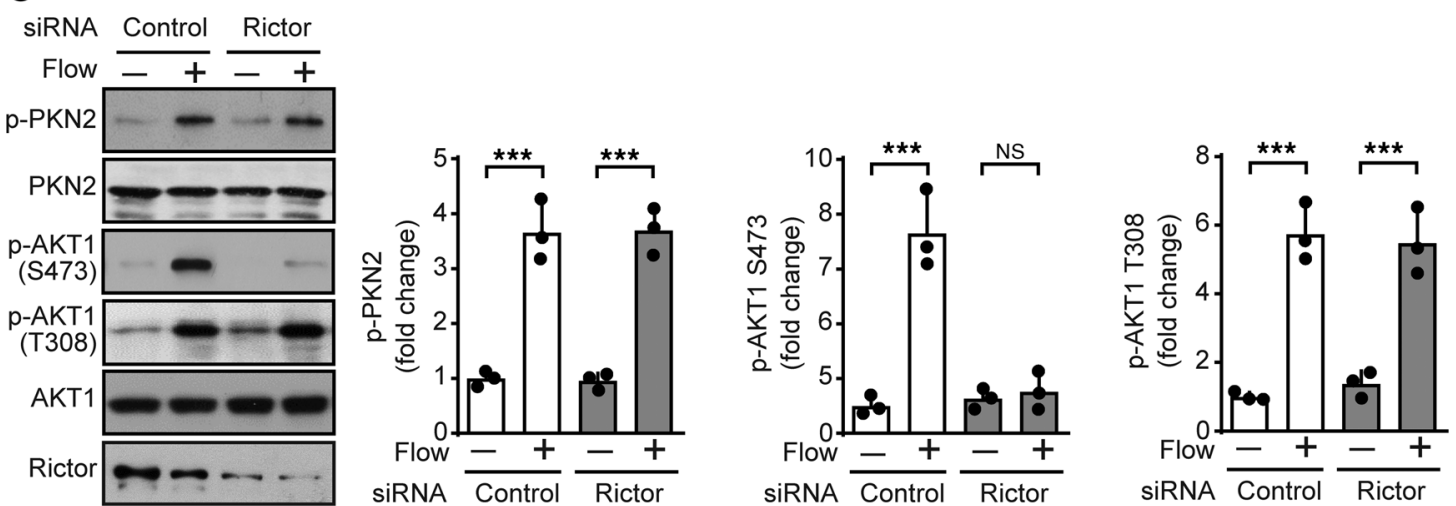

D
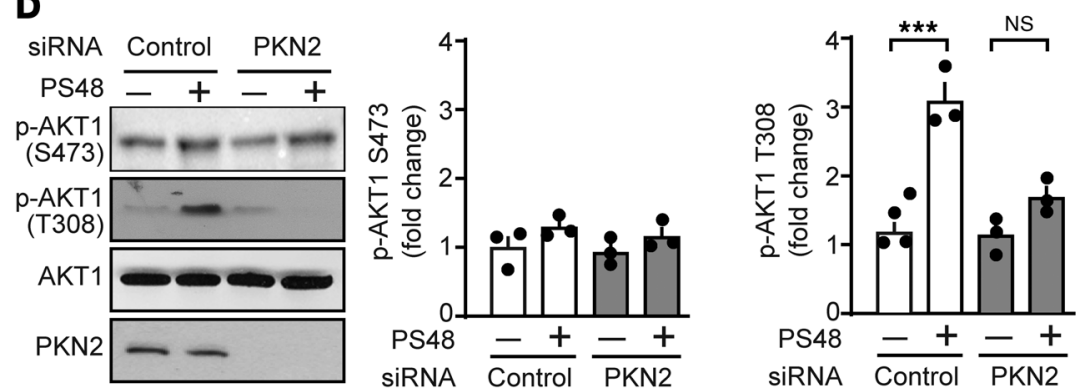

E

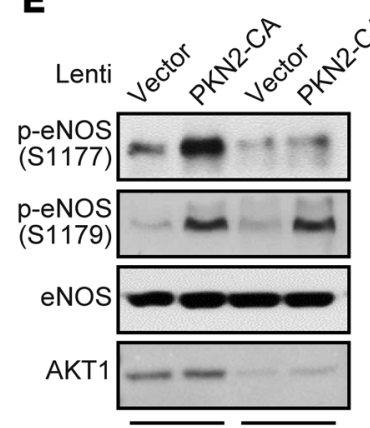

siRNA $\overline{\text { Control }} \overline{\text { AKT1 }}$
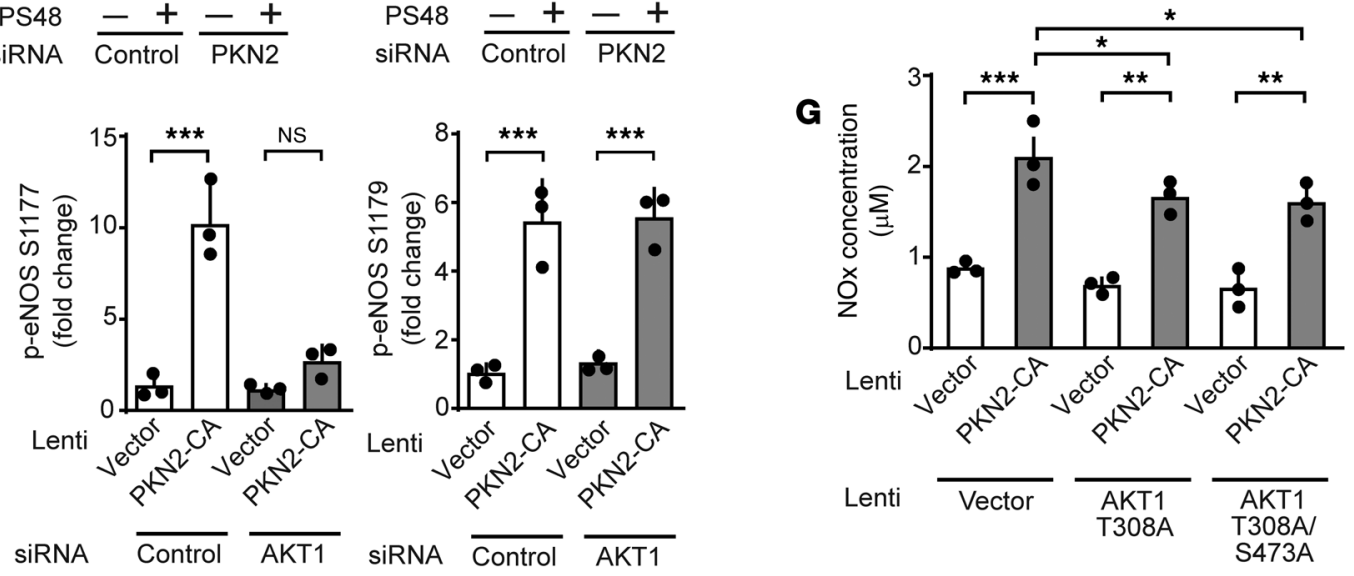
Figure 5. PKN2 mediates phosphorylation of AKT at threonine 308 but not at serine 473. (A-C) HUAECs were transfected with a control siRNA or siRNAs directed against PKN2 (A and D-G), PDK1 (B), Rictor (C), or AKT1 (E-G) and were then exposed to laminar flow $\left(15\right.$ dynes $\left./ \mathrm{cm}^{2}\right)$ for 15 minutes or for the indicated durations (A-C) or to $5 \mu \mathrm{M}$ PS48 for 15 minutes (D). Alternatively, a constitutively active mutant of PKN2 (PKN-CA) (E-G) as well as the indicated phosphosite mutants of AKT1 (C) were expressed by lentiviral transduction after siRNA-mediated knockdown of AKT1 (E and $\mathbf{F}$ ) in HUAECs. The levels of phosphorylated or total proteins was determined in lysates by immunoblotting (A-E), and NOx concentrations were measured in the supernatant (F and $\mathbf{G})$. Graphs in $\mathbf{A}-\mathbf{E}$ show the densitometric evaluation of the blots ( $n=3$ independent experiments). Control immunoblots for PKN2 and PDK1-knockdown efficiency in $\mathbf{A}, \mathbf{B}$, and $\mathbf{D}$ are from the same experiments as those shown in Figure $2 \mathrm{~B}$ and Figure $3, \mathrm{~A}$ and C, respectively. Data represent the mean $\pm \mathrm{SEM}$. ${ }^{*} P \leq 0.05,{ }^{*} P \leq 0.01$, and ${ }^{* *} P \leq 0.001$, by 1-way ANOVA with Tukey's post hoc test (A-F) and 2-way ANOVA with Bonferroni's post hoc test (G).

phorylation of eNOS, as well as through an independent pathway involving $\mathrm{Ca}^{2+}$, PKD1, and PKN2, resulting in phosphorylation of the serine residue serine 1179 of human eNOS. We found that PKN2 also plays an important role in serine 1177 phosphorylation, as it phosphorylated AKT at threonine 308 in response to flow and thereby promoted AKT-dependent serine 1177 phosphorylation of human eNOS. Thus, our data favor a model in which PKN2 integrates different flow-induced signaling pathways to link them to phosphorylation-dependent eNOS activation. It will be interesting to see whether the PKN2-mediated endothelial signaling process is affected in pathological situations in which eNOS activity is impaired, and whether PKN2 is also involved in other flow-independent mechanisms of eNOS activity regulation.

\section{Methods}

Reagents. Acetylcholine, phenylephrine, sodium nitroprusside (SNP), BAPTA-AM, PS48, ionomycin, KN93, wortmannin, LY294002, Ki8751, and tamoxifen were purchased from MilliporeSigma. YM-254890 was purchased from FUJIFILM Wako Chemicals (catalog 257-00631). Yoda1 was obtained from Maybridge (catalog SPB07298). Antibodies directed against phosphorylated eNOS (p-eNOS) (mouse S1176, human S1177, and bovine S1179; catalog 9571), p-AKT (S473; catalog 4060), p-AKT (T308; catalog 13038), AKT (catalog 9272), p-PDK1 (S241; catalog 3438), PDK1 (catalog 5662), p-PKN2 (T816; catalog 2611), PKN2 (catalog 2612), RhoA (catalog 2117), Rictor (catalog 2114), GAPDH (catalog 2118), and tubulin (catalog 2521S) were obtained from Cell Signaling Technology. Antibodies directed against p-eNOS (mouse S1178, human S1179, and bovine S1181; catalog PA564532) were obtained from Thermo Fisher Scientific. Anti-eNOS (catalog 610296) and anti-PKN1 (catalog 610687) antibodies were purchased from BD Biosciences. Anti-P2Y 2 antibody was obtained from Alomone Labs (catalog APR-010). Anti-Piezo1 antibody was purchased from Proteintech (catalog 15939-1-AP).

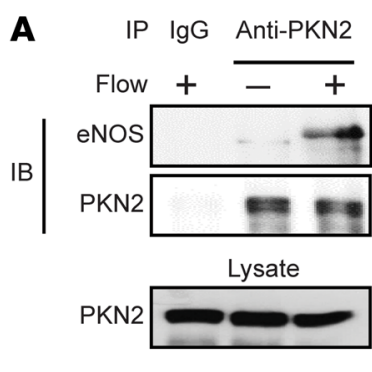

C

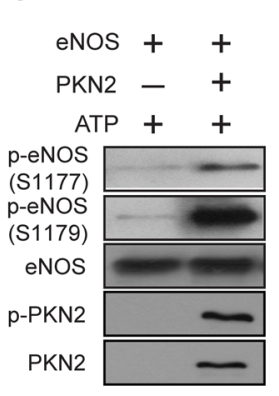

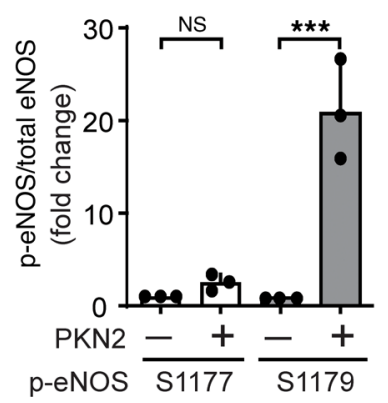
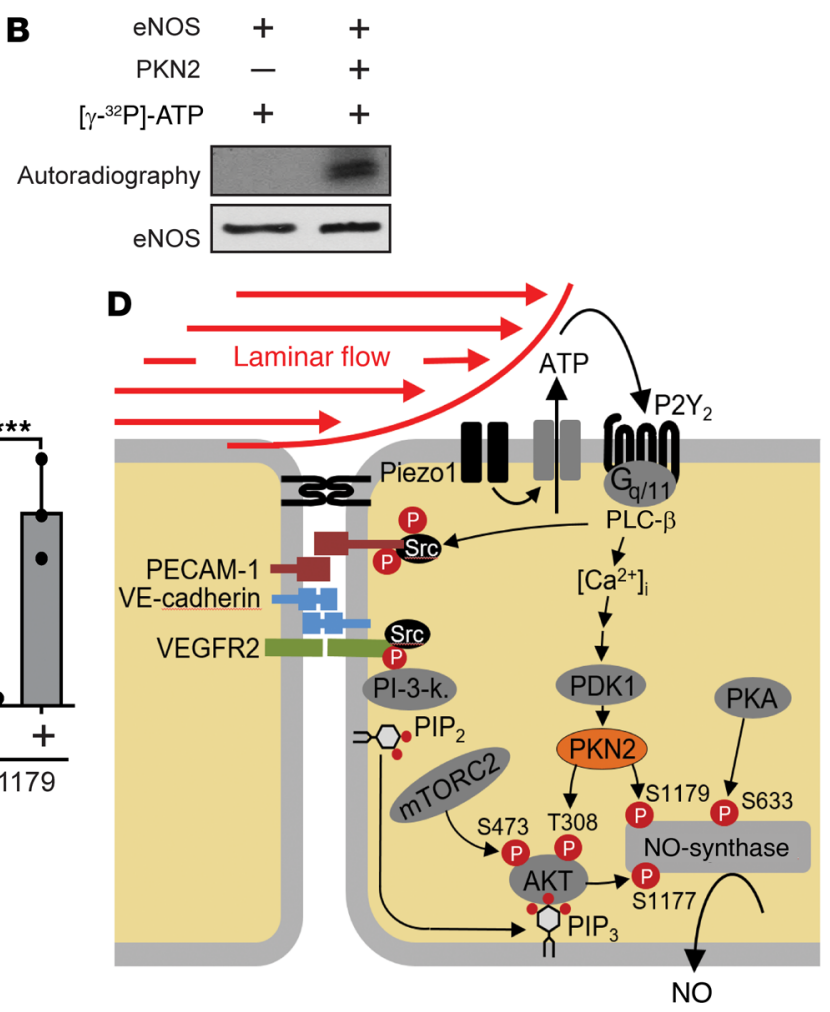

Figure 6. PKN2 mediates eNOS activation by direct phosphorylation at serine 1179 and by AKT-dependent phosphorylation at serine 1177. (A) HUAECs kept untreated or exposed to flow were lysed, and PKN2 was immunoprecipitated with a specific antibody. IgG served as a negative control. Shown are immunoblots (IB) of the lysate and the immunoprecipitate using anti-eNOS and anti-PKN2 antibodies. (B and C) Purified eNOS was incubated in kinase buffer with or without recombinant purified PKN2, together with $50 \mu \mathrm{M}[\gamma-32 \mathrm{P}]-\mathrm{ATP}(3000 \mathrm{Ci} / \mathrm{mmol} / \mathrm{L})$ (B) or ATP (C) for 30 minutes at $30^{\circ} \mathrm{C}$. Thereafter, samples were separated by SDS-PACE. Shown are an autoradiogram and a Coomassie stain of the gel (B). Alternatively, immunoblot analysis was performed using antibodies against eNOS and PKN2 proteins or eNOS S1177 and S1179, as well as the PKN2 T816 phosphosites (C). (D) Model of the role of PKN2 in flow-induced phosphorylation of eNOS. 
Primary cells. HUAECs and BAECs were purchased from Provitro AG and cultured in EGM-2 MV medium (Lonza). RFL-6 rat fibroblast cells (CCL-192) were purchased from the American Type Culture Collection (ATCC) and cultured in F-12 Nutrient Mixture (Ham, Invitrogen, Thermo Fisher Scientific) containing 10\% FCS.

siRNA-mediated knockdown. Endothelial cells were transfected with siRNA using Opti-MEM (Thermo Fisher Scientific) and Lipofectamine RNAiMAX (Invitrogen, Thermo Fisher Scientific) as described previously (16). The siRNAs used for screening consisted of pools of 3 siRNAs against nonreceptor tyrosine kinase, which show high expression in HUAECs and BAECs and were obtained from MilliporeSigma. The targeted sequences are shown in Supplemental Table 1. Other siRNAs used were against the following sequences: 5 '-GTGTTTGGTCTCAAGGACT-3' and 5'-GTGTCTACTTCCTGCTCTT-3 ' (Piezo1); and 5'-CCCTTCAGCACGGTGCTCT- ${ }^{\prime}$ and 5'-CTGCCTAGGGCCAAGCGCA-3' (P2RY2). The PDK1 endoribonuclease-prepared siRNA (esiRNA) (EHU079981), Rictor esiRNA (EHU076781), and control siRNAs (SIC001) were purchased from MilliporeSigma.

Shear stress assays. Endothelial cells were seeded onto $\mu$-Slide I Luer (catalog 80176, Ibidi), and laminar flow was applied on confluent monolayers using the Ibidi pump system chamber. The constant wall shear stress $(\tau)$ is given by: $\tau=\eta \times 131.6 \varphi$, where $\eta$ is the viscosity of the medium and $\varphi$ is the flow rate (according to the manufacturer's instructions).

Western blotting. Endothelial cells and mesenteric arteries were lysed in RIPA buffer freshly supplemented with protease and phosphatase inhibitors (PhosSTOP, Roche). Total cell lysates were subjected to SDSPAGE electrophoresis and transferred onto PVDF membranes. After blocking with $5 \%$ BSA for 1 hour, the membranes were incubated with primary antibodies overnight at $4^{\circ} \mathrm{C}$. The membranes were incubated with HRP-conjugated secondary antibodies for 1 hour at room temperature (Cell Signaling Technology; 1:3000 dilution) followed by chemiluminescence detection using ECL substrate (Pierce, Thermo Fisher Scientific) according to the manufacturer's protocol. The intensities of protein bands were quantitated using the ImageJ Gel Analysis program (NIH).

In vitro kinase and phosphorylation assay. Kinase activity was determined by performing an in vitro kinase assay (61). Briefly, endothelial cells were lysed in RIPA buffer containing protease inhibitors (cOmplete Protease Inhibitor Cocktail, Roche) and phosphatase inhibitors (PhosSTOP, Roche), and after centrifugation $(30,000 \mathrm{~g}, 15$ minutes), the supernatant was incubated with $3 \mu \mathrm{g}$ anti-PKN2 antibody overnight at $4^{\circ} \mathrm{C}$ on a rotating platform. Thereafter, $30 \mu \mathrm{L}$ protein $\mathrm{A} / \mathrm{G}$ Sepharose beads (Santa Cruz Biotechnology) preequilibrated in RIPA buffer were added and incubated for 1 hour at $4^{\circ} \mathrm{C}$. A/G Sepharose beads were then collected by centrifugation at $3500 \mathrm{~g}$ for 1 minute, washed 3 times, and resuspended in washing buffer (2O mM Tris-HCl [pH7.4], $10 \mathrm{mM} \mathrm{MgCl} 2)$. Pellets were resuspended in $20 \mu \mathrm{L}$ kinase buffer $(20$ mM HEPES [pH 7.4], 10 mM MgAc, 1 mM dithiothreitol, $50 \mu \mathrm{M}$ ATP, $20 \mu \mathrm{Ci}\left[\gamma^{-32} \mathrm{P}\right]$-ATP (Hartmann Analytic), together with $1 \mu \mathrm{g}$ MBP (catalog C1416, Santa Cruz Biotechnology) as a substrate for 30 minutes at room temperature. To stop the reaction, SDS-PAGE loading buffer was added. The reaction mixture was separated by SDS-PAGE, and ${ }^{32} \mathrm{P}-\mathrm{la}-$ beled proteins were visualized by autoradiography. For in vitro eNOS phosphorylation by PKN2, purified full-length human eNOS (catalog ALX-201.853.0010, Enzo Life Sciences) and PKN2 (P71-10G-05, SignalChem) were mixed and incubated for 30 minutes at $30^{\circ} \mathrm{C}$ with 10 $\mu \mathrm{Ci}\left[\gamma^{-32} \mathrm{P}\right]$-ATP in $20 \mu \mathrm{L}$ kinase buffer. All reactions were terminated by adding SDS-PAGE loading buffer. Proteins were separated by SDS-
PAGE, and the gels were stained with Coomassie. The radioactivity was analyzed by autoradiography. Alternatively, in vitro eNOS phosphorylation by PKN2 was detected by immunoblotting using the antip-eNOS S1179 antibody.

Animal models. All mice were backcrossed onto a C57BL/6N background at least 8-10 times, and experiments were performed with littermates as controls. Male and female animals (8-12 weeks old) were used unless stated otherwise. Mice were housed under a 12-hour light/12-hour dark cycle, with free access to food and water and under specific pathogen-free conditions. The endothelium-specific Cre transgenic lines Tek-CreERT2 and Cdh5-CreERT2 have been described previously $(62,63)$. Embryonic stem (ES) cells heterozygous for a targeted allele of $P k n 1$ or $P k n 2$, which, after removal of a cassette flanked by FRT sites, allows for Cre-mediated recombination, were purchased from the Knockout Mouse Project (KOMP) repository (UCD, Davis, California, USA). ES cell clones were injected into C57BL/6 blastocysts and transferred into pseudopregnant females. Chimeric offspring were bred with C57BL/6 mice to produce heterozygous animals. The germline transmission was confirmed in the F1 generation using a PCR genotyping strategy. In order to remove the selection cassette, mice were crossed with Flp-deleter mice (64) and subsequently crossed with Tek-CreERT2 and Cdh5-CreERT2 mice to obtain animals with inducible endothelium-specific deficiency (EC-Pkn1-KO or EC-Pkn2-KO mice). NOS $^{-/-}$mice (B6.129P2-Nos3tm1Unc/1) were obtained from The Jackson Laboratory.

Determination of NOx and cyclic GMP levels. NOx levels were measured as described previously (12). In brief, blood collected by venous puncture was immediately centrifuged at $6800 \mathrm{~g}$ for 5 minutes at $4^{\circ} \mathrm{C}$, and $20 \mu \mathrm{L}$ plasma was used for nitrate measurement. For the experiment to determine flow-induced nitrate release from endothelial cells, cells were plated on culture glass of the BioTech-Flow viscometer chambers (MOS Technologies). Cells were starved in serum-free medium for 6 hours and exposed to laminar flow for 30 minutes. NOx production was measured according to NOx accumulation in the medium and plasma using a NOx fluorometric assay kit from Cayman Chemical according to the manufacturer's instructions.

Cyclic GMP (cGMP) levels were measured using the RFL-6 reporter cell assay as described previously (65). RFL-6 cells were grown to confluence in 6-well plates and washed twice in Locke's solution (154 mM NaCl, $5.6 \mathrm{mM} \mathrm{KCl}, 2 \mathrm{mM} \mathrm{CaCl}_{2}, 1 \mathrm{mM} \mathrm{MgCl}_{2}$, and 10 $\mathrm{mM}$ HEPES) containing $0.3 \mathrm{mM}$ 3-isobutyl-1-methylxanthine (IBMX) before the experiment. RFL-6 cells were incubated with supernatants of HUAECs expressing control vector, WT eNOS, or mutants of eNOS in the absence or presence of L-NAME $(1 \mu \mathrm{M})$. Thereafter, the incubation was stopped by the addition of ice-cold $50 \mathrm{mM}$ sodium acetate buffer ( $\mathrm{pH}$ 4.0). The cells were then lysed and the cGMP concentration was determined using a cGMP assay kit according to the manufacturer's instructions (Abcam, catalog ab65356).

Wire myography. Two weeks after the last tamoxifen injection, mice were sacrificed by $\mathrm{CO}_{2}$ inhalation. Intact first-order mesenteric arteries $(2 \mathrm{~mm})$ were isolated from the mesentery and mounted onto a conventional myograph setup (610-M, Danish Myo Technology) and kept in Krebs solution (118.1 mM NaCl, $4.8 \mathrm{mM} \mathrm{KCl}, 2.5 \mathrm{mM} \mathrm{CaCl}_{2}, 1.2 \mathrm{mM}$ $\mathrm{MgSO}_{4}, 1.2 \mathrm{mM} \mathrm{KH}_{2} \mathrm{PO}_{4}, 25 \mathrm{mM} \mathrm{NaHCO}_{3}, 9.3 \mathrm{mM}$ glucose, and 0.026 mM EDTA). Arterial segments were distended to the diameter at which maximal contractile responses to $10 \mu \mathrm{M}$ phenylephrine were observed (66). After a 30-minute recovery period of normalization, the contrac- 
A

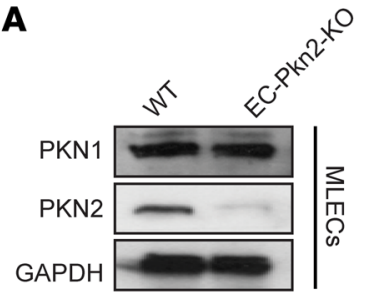

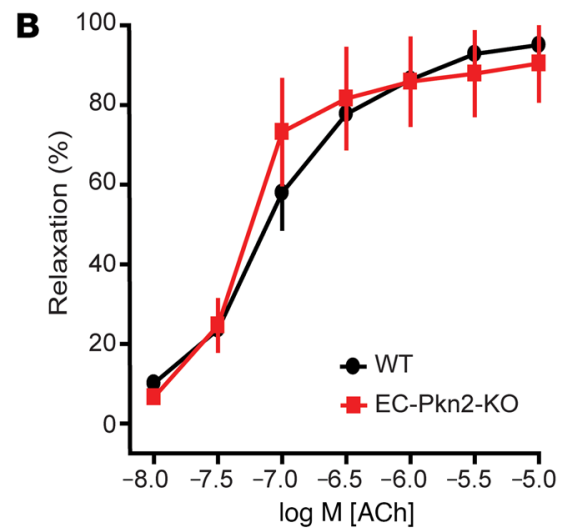

$\log \mathrm{M}[\mathrm{ACh}]$

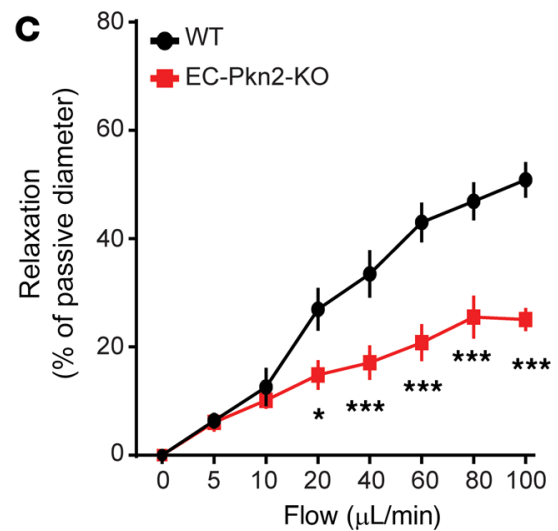

D
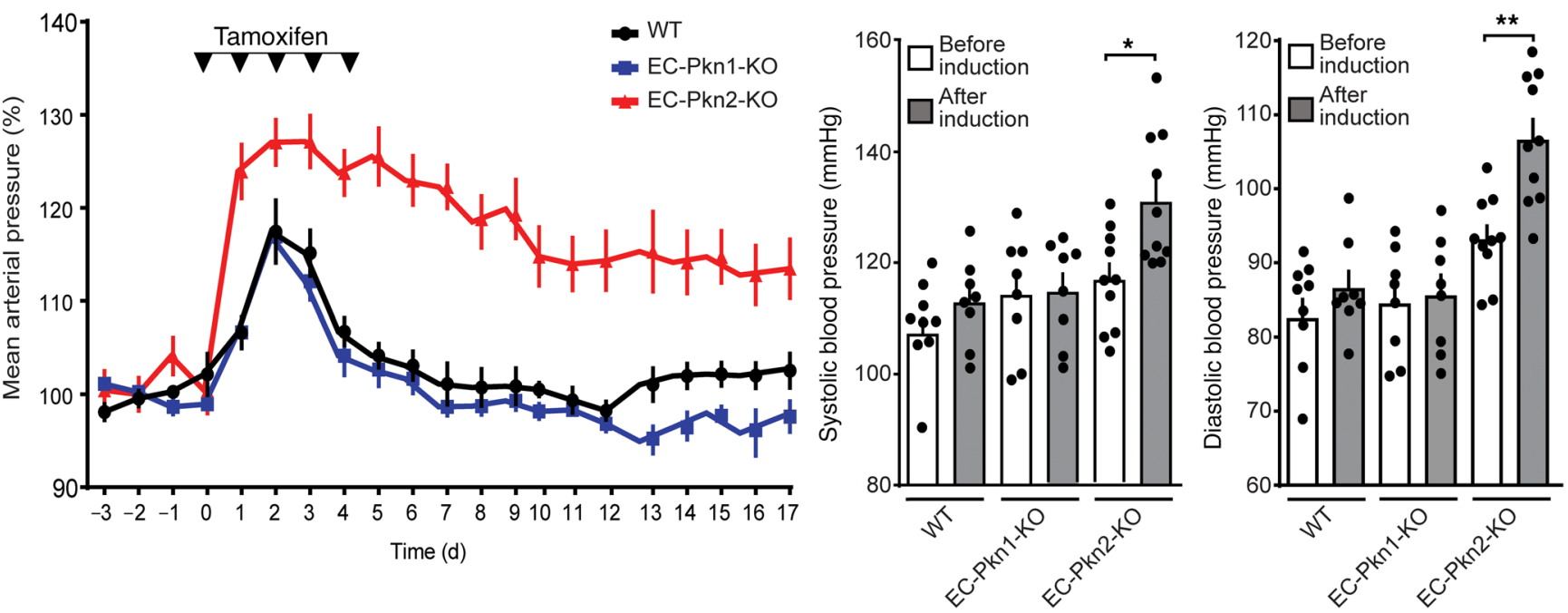

$\mathbf{E}$

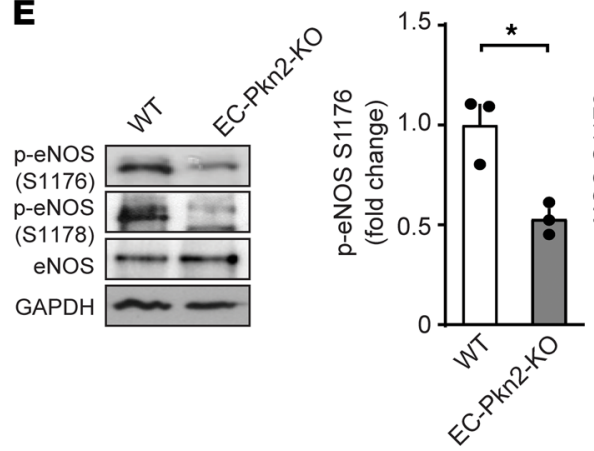

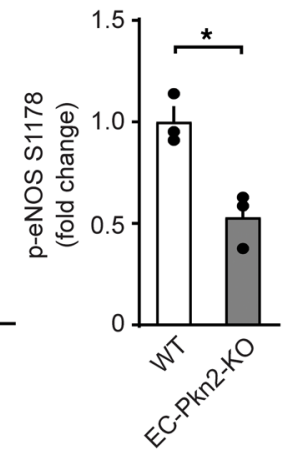

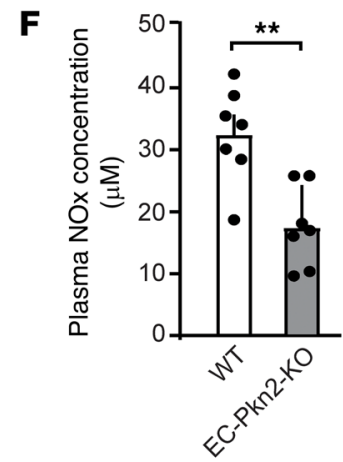

Figure 7. Endothelial PKN2 deficiency results in loss of flow-induced vasodilation and arterial hypertension. (A) Immunoblot analysis of PKN1 and PKN2 expression in mouse lung endothelial cells (MLECs) from 8-week-old WT and induced EC-Pkn2-KO mice. (B) Effect of acetylcholine (ACh) on the tone of mesenteric artery stripes from WT $(n=7)$ and EC-Pkn2-KO animals $(n=7)$ was determined after precontraction with $10 \mu \mathrm{M}$ phenylephrine. (C) Mesenteric arteries isolated from tamoxifen-treated WT or EC-Pkn2-KO mice were precontracted with $100 \mathrm{nM}$ of the thromboxane $\mathrm{A}_{2}$ analog U46619 and were then exposed to stepwise increases in perfusion flow. Flow-induced vasorelaxation is shown as a percentage of the passive vessel diameter ( $n=7$ WT and EC-Pkn2-KO mice). (D) Blood pressure in WT $(n=12)$, EC-Pkn1-KO mice $(n=8)$, and EC-Pkn2-KO ( $n=12)$ animals before, during, and after tamoxifen treatment (induction). Average blood pressure 3 days before treatment was set at $100 \%$. Graphs show systolic and diastolic arterial blood pressure 4 days before tamoxifen treatment and in the second week after treatment. (E) eNOS phosphorylation at S1176 and S1178 in lysates from mesenteric arteries prepared from tamoxifen-treated WT and EC-Pkn2-KO mice ( $n=3$ animals). (F) Plasma nitrate and NOx levels in WT $(n=7)$ and EC-Pkn2-KO mice $(n=7) 10$ days after treatment. Data represent the mean \pm SEM. ${ }^{*} P \leq 0.05$, ${ }^{* *} P \leq 0.01$, and ${ }^{* * *} P \leq 0.001$, by 2-way ANOVA with Bonferroni's post hoc test (B and $\mathbf{C}$ ) and paired, 2-tailed Student's $t$ test (D) or unpaired, 2-tailed Student's $t$ test (F).

tile and relaxation responses to the indicated agonists were tested. All arterial segments were allowed 30 minutes of recovery after normalization. Endothelial integrity was checked, and the segments that showed less than $80 \%$ relaxation were excluded from the experiment.
Pressure myography. Pressure myography experiments were performed as described previously (16). In brief, 10-14 days after tamoxifen injection, second-order mesenteric arteries were removed from the mesentery and mounted between 2 glass micro- 
pipettes seated in a pressure myograph chamber (114P, Danish Myo Technology). The temperature of the chamber was kept at a constant $37^{\circ} \mathrm{C}$ during the experiment. The mounted artery was initially pressurized to $20 \mathrm{mmHg}$ under no-flow conditions and incubated for 30 minutes. Pressure was then increased to $60 \mathrm{mmHg}$, and the vessel was incubated for 10 minutes to allow it to reach a steadystate diameter. Arterial segments were contracted with 50-150 nM $\mathrm{U} 46619$ to $40 \%-50 \%$ of the passive diameter. After reaching a stable baseline, flow was increased in a stepwise manner by changing the pressure of the inflow and outflow sides inversely, thereby creating a pressure difference inside the lumen of the artery without altering the intraluminal pressure. The viability of the vessel was verified at the end of the experiment by dilating the vessel with acetylcholine $(10 \mu \mathrm{M})$. Arteries showing less than $60 \%$ relaxation were considered damaged and were omitted from further analysis. Vasodilatation to flow was calculated as the percentage of the U46619-induced contraction, as described previously (67), using the following equation: percentage of relaxation $=100 \times\left(\mathrm{D}_{\mathrm{F}}-\right.$ $\left.\mathrm{DU}_{46619}\right) /\left(\mathrm{DP}_{\mathrm{D}}-\mathrm{DU}_{46619}\right)$, where $\mathrm{D}$ represents the external diameter of the vessels; $\mathrm{D}_{\mathrm{F}}$ is the vessel diameter during flow; $\mathrm{DU}_{46619}$ is the diameter after $\mathrm{U} 46619$ contraction; and $\mathrm{DP}_{\mathrm{D}}$ is the passive diameter without any treatment.

Telemetric blood pressure measurements. Blood pressure measurements were performed in conscious, unrestrained mice with a radiotelemetry system (PA-C10, Data Sciences International) as described previously (12). Mice were caged with blinded identity and in random order.

Lentiviral infection of cells. Human WT or mutant forms of eNOS, AKT1, and PKN2 were cloned into the lentiviral pLVX-IRES-ZsGreen1 expression vector (Clontech) and transfected into HEK293T cells along with the envelope plasmid pMD2.G and the packaging plasmid psPAX2 using Lipofectamine 3000 Transfection Reagent (Thermo Fisher Scientific) according to the manufacturer's protocol. After 48 hours, supernatants containing lentiviral particles were harvested and filtered through a $0.45 \mu \mathrm{m}$ low-protein-binding Durapore membrane (Millex) to remove cell debris. HUAECs were previously transfected with an siRNA against human eNOS or AKT using OptiMEM and Lipofectamine RNAiMAX (Invitrogen, Thermo Fisher Scientific). For lentiviral transduction, HUAECs were seeded in 6-well plates, and the concentrated lentivirus was added. After 48 hours, the cells were used for further analyses.

Adeno-associated virus infection. AAV2-QuadYF carrying the cDNA encoding eNOS WT, the S1177A and S1179A mutants of eNOS, as well as EGFP were generated by VectorBuilder (Cyagen Biosciences). Tento 12-week-old eNOS $^{-/-}$mice were anesthetized by isofluorane inhalation. Mice were given AAV2-QuadYF-eNOS WT, S1177A, S1179A, or AAV2-QuadYF-EGFP $\left(1 \times 10^{11}\right.$ viral genomes in $100 \mu \mathrm{L}$ saline $)$ by intravenous injection. One week after virus injection, the carotid arteries were harvested to measure gene expression and flow-induced vasorelaxation using a pressure myography system.

Expression analysis. Total RNA was isolated using an RNeasy Micro Kit (QIAGEN) according to the manufacturer's instructions. Reverse transcription (RT) was performed using the ProtoScript II Reverse Transcription kit (M0368S, New England BioLabs). The resulting cDNA was used as a template for quantitative PCR (qPCR) reactions using the LightCycler 480 Probe Master System (Roche) following the manufacturer's protocol. The primer sequences are listed in Supplemental Table 2. The resulting quantification cycle $(\mathrm{Cq})$ values were analyzed and normalized to the reference gene GAPDH.

Proteomic and phosphoproteomic analysis. Samples were prepared as described earlier (12), with modifications. Cells were lysed in $4 \%$ SDS and $100 \mathrm{mM}$ Tris ( $\mathrm{pH}$ 7.6) without a protease inhibitor by 10 minutes of boiling followed by sonication prior to the removal of cell debris by centrifugation. Further, samples were labeled using reductive dimethylation as reported previously (68), and STop And Go Extraction (STAGE) tipping of the proteomic samples was omitted. Using MARMoSET (69), liquid chromatography-tandem mass spectrometry (LC-MS/MS) instrumentation, parameters were extracted. Peptide/spectrum matching and quantitation were performed using the MaxQuant suite of algorithms $(70,71)$ against the UniProt (72) mouse canonical and bovine isoforms database (downloaded on August 19, 2019; 46,707 entries). Intensity values of the phosphosites shown were normalized to eNOS intensity and thus protein abundance in the proteome.

Statistics. All statistical analysis was performed using GraphPad Prism, version 6.07 (GraphPad Software). All experimental values are presented as the mean \pm SEM. Statistical significance was tested using either ANOVA or an unpaired Student's $t$ test. Statistical analysis comparing 2 groups was performed with an unpaired, 2-tailed Student's $t$ test, multiple group comparisons were analyzed with 1-way ANOVA followed by Tukey's post hoc test, and comparisons between multiple experimental groups at different time points were performed using 2-way ANOVA followed by Bonferroni's post hoc test. A $P$ value of less than 0.05 was considered statistically significant.

Study approval. All procedures involving animal care and use in this study were approved by the local animal ethics committees (Regierungspräsidium Darmstadt, Germany).

\section{Author contributions}

YJJ performed most experiments, designed the study, analyzed and discussed data, and contributed to the writing of the manuscript. RC, RL, GL, and SPW assisted with the myography experiments and blood pressure measurement. AI and JG performed the phosphoproteomic analysis. NW supervised the study, discussed data, and commented on the manuscript. SO initiated and supervised the study, discussed data, and wrote the manuscript. All authors read and commented on the manuscript.

\section{Acknowledgments}

We thank Svea Hümmer for secretarial help and Ulrike Krüger, Claudia Ullmann, Martina Finkbeiner, and Sylvia Jeratsch for expert technical support. This work was supported by the Collaborative Research Center 834 of the German Research Foundation, the Hungarian Academy of Sciences Premium Postdoctoral Research Program (PPD2019-20/2019-439 to AI), and an Alexander von Humboldt Foundation fellowship (to YJJ).

Address correspondence to: Young-June Jin or Stefan Offermanns, Department of Pharmacology, Max Planck Institute for Heart and Lung Research, Ludwigstrasse 43, 61231 Bad Nauheim, Germany. Phone: 48.6032.705.1268; Email: young-june.jin@mpi-bn.mpg. de (YYJ); Phone: 49.6032.705.1201; Email: stefan.offermanns@ mpi-bn.mpg.de (SF). 
1. Zhao Y, et al. Vascular nitric oxide: beyond eNOS. JPharmacol Sci. 2015;129(2):83-94.

2. Siragusa M, Fleming I. The eNOS signalosome and its link to endothelial dysfunction. Pflugers Arch. 2016;468(7):1125-1137.

3. Garcia V, Sessa WC. Endothelial NOS: perspective and recent developments. Br J Pharmacol. 2019;176(2):189-196.

4. Fleming I. Molecular mechanisms underlying the activation of eNOS. Pflugers Arch. 2010;459(6):793-806.

5. Balligand JL, et al. eNOS activation by physical forces: from short-term regulation of contraction to chronic remodeling of cardiovascular tissues. Physiol Rev. 2009;89(2):481-534.

6. Fulton D, et al. Regulation of endothelium-derived nitric oxide production by the protein kinase Akt. Nature. 1999;399(6736):597-601.

7. Dimmeler S, et al. Activation of nitric oxide synthase in endothelial cells by Akt-dependent phosphorylation. Nature. 1999;399(6736):601-605.

8. Boo YC, et al. Shear stress stimulates phosphorylation of eNOS at Ser(635) by a protein kinase A-dependent mechanism. Am J Physiol Heart Circ Physiol. 2002;283(5):H1819-H1828.

9. Qian J, Fulton D. Post-translational regulation of endothelial nitric oxide synthase in vascular endothelium. Front Physiol. 2013;4:347.

10. Bauer PM, et al. Compensatory phosphorylation and protein-protein interactions revealed by loss of function and gain of function mutants of multiple serine phosphorylation sites in endothelial nitric-oxide synthase. J Biol Chem. 2003;278(17):14841-14849.

11. Michell BJ, et al. Identification of regulatory sites of phosphorylation of the bovine endothelial nitric-oxide synthase at serine 617 and serine 635. J Biol Chem. 2002;277(44):42344-42351.

12. Iring A, et al. Shear stress-induced endothelial adrenomedullin signaling regulates vascular tone and blood pressure. J Clin Invest. 2019;130(7):2775-2791.

13. Boo YC, et al. Shear stress stimulates phosphorylation of endothelial nitric-oxide synthase at Ser1179 by Akt-independent mechanisms: role of protein kinase A. J Biol Chem. 2002;277(5):3388-3396.

14. Zhang Y, et al. AMP-activated protein kinase is involved in endothelial NO synthase activation in response to shear stress. Arterioscler Thromb Vasc Biol. 2006;26(6):1281-1287.

15. Wang S, et al. Endothelial cation channel PIEZO1 controls blood pressure by mediating flow-induced ATP release. J Clin Invest. 2016;126(12):4527-4536.

16. Wang S, et al. P2Y and $G q / G_{11}$ control blood pressure by mediating endothelial mechanotransduction. JClin Invest. 2015;125(8):3077-3086.

17. Koo A, et al. In silico modeling of shear-stressinduced nitric oxide production in endothelial cells through systems biology. Biophys $J$. 2013;104(10):2295-2306

18. Lee MY, et al. Endothelial cell autonomous role of Akt1: regulation of vascular tone and ischemia-induced arteriogenesis. Arterioscler Thromb Vasc Biol. 2018;38(4):870-879.

19. Manning BD, Toker A. AKT/PKB signaling: navigating the network. Cell. 2017;169(3):381-405.

20. Hers I, et al. Akt signalling in health and disease.
Cell Signal. 2011;23(10):1515-1527.

21. Marrocco V, et al. PKC and PKN in heart disease. JMol Cell Cardiol. 2019;128:212-226.

22. Thumkeo D, et al. Physiological roles of Rho and Rho effectors in mammals. Eur J Cell Biol. 2013;92(10-11):303-315.

23. Watanabe G, et al. Protein kinase N (PKN) and PKN-related protein rhophilin as targets of small GTPase Rho. Science. 1996;271(5249):645-648.

24. Hutchinson CL, et al. Differential binding of RhoA, RhoB, and RhoC to protein kinase C-related kinase (PRK) isoforms PRK1, PRK2, and PRK3: PRKs have the highest affinity for RhoB. Biochemistry. 2013;52(45):7999-8011.

25. Amano M, et al. Identification of a putative target for Rho as the serine-threonine kinase protein kinase N. Science. 1996;271(5249):648-650.

26. Dettori R, et al. Regulation of the interaction between protein kinase $\mathrm{C}$-related protein kinase 2 (PRK2) and its upstream kinase, 3-phosphoinositide-dependent protein kinase 1 (PDK1). J Biol Chem. 2009;284(44):30318-30327.

27. Balendran A, et al. A 3-phosphoinositide-dependent protein kinase-1 (PDK1) docking site is required for the phosphorylation of protein kinase Czeta (PKCzeta) and PKC-related kinase 2 by PDK1. J Biol Chem. 2000;275(27):20806-20813.

28. Vincent S, Settleman J. The PRK2 kinase is a potential effector target of both Rho and Rac GTPases and regulates actin cytoskeletal organization. Mol Cell Biol. 1997;17(4):2247-2256.

29. Lachmann S, et al. Regulatory domain selectivity in the cell-type specific PKN-dependence of cell migration. PLoS One. 2011;6(7):e21732.

30. Bourguignon LY, et al. Hyaluronan-CD44 interaction with Rac1-dependent protein kinase $\mathrm{N}$-gamma promotes phospholipase Cgamma1 activation, $\mathrm{Ca}(2+)$ signaling, and cortactin-cytoskeleton function leading to keratinocyte adhesion and differentiation. JBiol Chem. 2004;279(28):29654-29669.

31. Calautti E, et al. Fyn tyrosine kinase is a downstream mediator of Rho/PRK2 function in keratinocyte cell-cell adhesion. JCell Biol. 2002;156(1):137-148.

32. Lee SJ, et al. PKN2 and Cdo interact to activate AKT and promote myoblast differentiation. Cell Death Dis. 2016;7(10):e2431.

33. Danno S, et al. PKN2 is essential for mouse embryonic development and proliferation of mouse fibroblasts. Genes Cells. 2017;22(2):220-236.

34. Quetier I, et al. Knockout of the PKN family of Rho effector kinases reveals a non-redundant role for PKN2 in developmental mesoderm expansion. Cell Rep. 2016;14(3):440-448.

35. Biondi RM, et al. Identification of a pocket in the PDK1 kinase domain that interacts with PIF and the C-terminal residues of PKA. EMBO J. 2000;19(5):979-988.

36. Takasaki J, et al. A novel Galphaq/11-selective inhibitor. J Biol Chem. 2004;279(46):47438-47445.

37. Tzima E, et al. A mechanosensory complex that mediates the endothelial cell response to fluid shear stress. Nature. 2005;437(7057):426-431.

38. Boo YC, et al. Endothelial NO synthase phosphorylated at SER635 produces NO without requiring intracellular calcium increase. Free Radic Biol Med. 2003;35(7):729-741.
39. Schleicher M, et al. The Akt1-eNOS axis illustrates the specificity of kinase-substrate relationships in vivo. Sci Signal. 2009;2(82):ra41.

40. Luo Z, et al. Acute modulation of endothelial Akt/PKB activity alters nitric oxide-dependent vasomotor activity in vivo. J Clin Invest. 2000;106(4):493-499.

41. Mukai Y, et al. Decreased vascular lesion formation in mice with inducible endothelial-specific expression of protein kinase Akt. J Clin Invest. 2006;116(2):334-343

42. Fayard E, et al. Protein kinase B (PKB/Akt), a key mediator of the PI3K signaling pathway. Curr Top Microbiol Immunol. 2010;346:31-56.

43. Go YM, et al. Phosphatidylinositol 3-kinase gamma mediates shear stress-dependent activation of JNK in endothelial cells. Am J Physiol. 1998;275(5):H1898-H1904.

44. Alessi DR, et al. Characterization of a 3-phosphoinositide-dependent protein kinase which phosphorylates and activates protein kinase Balpha. Curr Biol. 1997;7(4):261-269.

45. Alessi DR, et al. Mechanism of activation of protein kinase B by insulin and IGF-1. EMBO J. 1996;15(23):6541-6551.

46. Sarbassov DD, et al. Phosphorylation and regulation of Akt/PKB by the rictor-mTOR complex. Science. 2005;307(5712):1098-1101.

47. Hresko RC, Mueckler M. mTOR.RICTOR is the Ser473 kinase for Akt/protein kinase B in 3T3-L1 adipocytes. J Biol Chem. 2005;280(49):40406-40416.

48. Koh H, et al. Inhibition of Akt and its anti-apoptotic activities by tumor necrosis factor-induced protein kinase C-related kinase 2 (PRK2) cleavage. J Biol Chem. 2000;275(44):34451-34458.

49. Patel H, et al. Novel roles of PRK1 and PRK2 in cilia and cancer biology. Sci Rep. 2020;10(1):3902.

50. Tzima E, et al. Activation of integrins in endothelial cells by fluid shear stress mediates Rho-dependent cytoskeletal alignment. EMBO J. 2001;20(17):4639-4647.

51. Xu S, et al. PECAM1 regulates flow-mediated Gab1 tyrosine phosphorylation and signaling. Cell Signal. 2016;28(3):117-124.

52. Taniyama Y, et al. Pyk2- and Src-dependent tyrosine phosphorylation of PDK1 regulates focal adhesions. Mol Cell Biol. 2003;23(22):8019-8029.

53. Gocher AM, et al. Akt activation by $\mathrm{Ca}(2+) /$ calmodulin-dependent protein kinase kinase 2 (CaMKK2) in ovarian cancer cells. J Biol Chem. 2017;292(34):14188-14204.

54. Kashiwagi S, et al. eNOS phosphorylation on serine 1176 affects insulin sensitivity and adiposity. Biochem Biophys Res Commun. 2013;431(2):284-290.

55 . Atochin DN, et al. The phosphorylation state of eNOS modulates vascular reactivity and outcome of cerebral ischemia in vivo. J Clin Invest. 2007;117(7):1961-1967.

56. Li Q, et al. Deficient eNOS phosphorylation is a mechanism for diabetic vascular dysfunction contributing to increased stroke size. Stroke. 2013;44(11):3183-3188.

57. Eroglu E, et al. Discordance between eNOS phosphorylation and activation revealed by multispectral imaging and chemogenetic methods. Proc Nat Acad Sci U S A. 2019;116(40):20210-20217. 
58. Salerno JC, et al. An autoinhibitory control element defines calcium-regulated isoforms of nitric oxide synthase. J Biol Chem. 1997;272(47):29769-29777.

59. McCabe TJ, et al. Enhanced electron flux and reduced calmodulin dissociation may explain "calcium-independent" eNOS activation by phosphorylation. J Biol Chem. 2000;275(9):6123-6128.

60. Lane P, Gross SS. Disabling a C-terminal autoinhibitory control element in endothelial nitric-oxide synthase by phosphorylation provides a molecular explanation for activation of vascular NO synthesis by diverse physiological stimuli. J Biol Chem. 2002;277(21):19087-19094.

61. Powell DW, et al. Identification of 14-3-3zeta as a protein kinase B/Akt substrate. J Biol Chem. 2002;277(24):21639-21642.

62. Korhonen H, et al. Anaphylactic shock depends on endothelial Gq/G11. JExp Med. 2009;206(2):411-420.

63. Sorensen I, et al. DLL1-mediated Notch activation regulates endothelial identity in mouse fetal arteries. Blood. 2009;113(22):5680-5688.

64. Farley FW, et al. Widespread recombinase expression using FLPeR (flipper) mice. Genesis. 2000;28(3-4):106-110.

65. Ishii K, et al. A simple and sensitive bioassay method for detection of EDRF with RFL-6 rat lung fibroblasts. Am J Physiol. 1991;261(2 pt 2):H598-H6O3.

66. Bailey RJ, et al. Pharmacological characterization of rat amylin receptors: implications for the identification of amylin receptor subtypes. Br J Pharmacol. 2012;166(1):151-167.

67. Liu C, et al. Depletion of intracellular $\mathrm{Ca}^{2+}$ stores enhances flow-induced vascular dilatation in rat small mesenteric artery. $\mathrm{BrJPharmacol}$. 2006;147(5):506-515.

68. Kim HT, et al. Myh10 deficiency leads to defective extracellular matrix remodeling and pulmonary disease. Nat Commun. 2018;9(1):4600.

69. Kiweler M, et al. MARMoSET - extracting publication-ready mass spectrometry metadata from RAW files. Mol Cell Proteomics. 2019;18(8):1700-1702.

70. Cox J, Mann M. MaxQuant enables high peptide identification rates, individualized p.p.b.-range mass accuracies and proteome-wide protein quantification. Nat Biotechnol. 2008;26(12):1367-1372.

71. Cox J, et al. Andromeda: a peptide search engine integrated into the MaxQuant environment. JProteome Res. 2011;10(4):1794-1805.

72. UniProt C. UniProt: a worldwide hub of protein knowledge. Nucleic Acids Res. 2019;47(d1):D506-D515. 